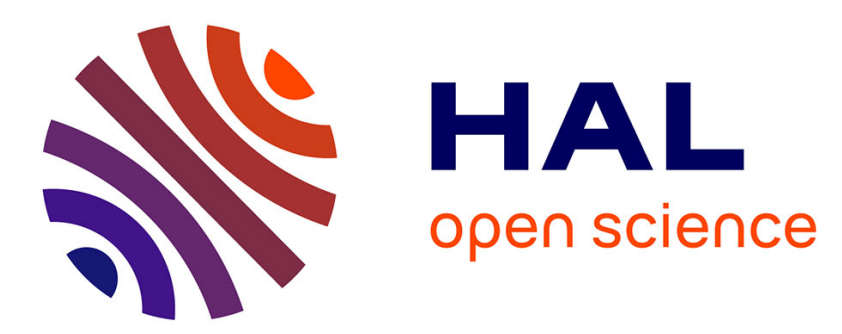

\title{
Open-channel flows through emergent rigid vegetation: Effects of bed roughness and shallowness on the flow structure and surface waves
}

M. Chatelain, S. Proust

\section{- To cite this version:}

M. Chatelain, S. Proust. Open-channel flows through emergent rigid vegetation: Effects of bed roughness and shallowness on the flow structure and surface waves. Physics of Fluids, 2021, 33 (10), pp.106602. 10.1063/5.0063288 . hal-03369211

\section{HAL Id: hal-03369211 \\ https://hal.science/hal-03369211}

Submitted on 7 Oct 2021

HAL is a multi-disciplinary open access archive for the deposit and dissemination of scientific research documents, whether they are published or not. The documents may come from teaching and research institutions in France or abroad, or from public or private research centers.
L'archive ouverte pluridisciplinaire HAL, est destinée au dépôt et à la diffusion de documents scientifiques de niveau recherche, publiés ou non, émanant des établissements d'enseignement et de recherche français ou étrangers, des laboratoires publics ou privés. 


\title{
Open-channel flows through emergent rigid vegetation: effects of bed roughness
}

\section{and shallowness on the flow structure and surface waves}

\author{
M. Chatelain ${ }^{1}$ and S. Proust ${ }^{1, \text { a) }}$
}

INRAE, UR RiverLy, Centre de Lyon-Villeurbanne, 5 rue de la Doua CS 20244, 69625 Villeurbanne, France

(Dated: 27 September 2021)

Free-surface flows through a staggered cylinder array were investigated in an open-channel flume. The cylinders simulated rigid emergent vegetation. Specifically, we studied four flow cases with a two-factor design comprising flow rate $\left(7\right.$ and $\left.131 \mathrm{~s}^{-1}\right)$ and bed-surface state (hydraulically-rough and -smooth). We have primarily assessed the effects of bed roughness and shallowness on the time-averaged flow structure and the transverse fluctuating flow motion in the cylinder-wake. Secondarily, the effects of the former on the vortex-shedding-induced surface waves were quantified. To gain further insight into the bed roughness effect on flow structure, we conducted transient flow simulations using a hybrid RANS (Reynolds-Averaged Navier-Stokes) - LES (Large-Eddy Simulation) turbulence model. For all cases, downstream of a cylinder, an upward flow occurs and two counter-rotating secondary current cells develop. The latter bring high-momentum fluid from the high-speed region into the cylinder-wake, resulting in a near-bed streamwise velocity-bulge. The measured upward flow and velocity-bulge are greater for the roughbed cases than for the smooth-bed cases. The simulated upward flow and velocity-bulge increase with an increasing roughness height, while secondary currents decay faster in the longitudinal direction. For the rough-bed cases, in the cylinder-wake, the transverse fluctuating flow motion is hindered by the rough-bed induced turbulence over the whole water column, irrespective of the shallowness level. Coupled with the fluctuating flow motion, we have observed for three flow cases noticeable surface oscillations (termed 'seiche waves'), whose amplitude decreases with decreasing flow depth. Under the combined effects of strong shallowness and a rough bed, seiche waves vanished.

${ }^{a)}$ Electronic mail: sebastien.proust@inrae.fr 


\section{INTRODUCTION}

Characterizing the structure of vegetated flows is an unavoidable step to better understand physical processes such as sediment transport, bed morphodynamics, and diffusion of contaminants and nutrients along rivers, in floodplain woodlands, marshes, and mangroves areas ${ }^{1,2}$. When simulating open-channel flows through rigid vegetation, circular cylinder arrays have often been used in physical modeling, with e.g., uniform arrays of aligned or staggered cylinders ${ }^{3-5}$, or randomly disposed cylinders ${ }^{6-8}$, open channels partially obstructed by a vegetation patch $^{9,10}$, or multi-layers vegetation models ${ }^{11}$.

The present paper reports an experimental and numerical study of flows through a staggered array of emergent circular cylinders (wooden stems), which have been installed over a hydraulicallysmooth bed (glass bottom) or hydraulically-rough bed (dense artificial grass). The study was first and foremost aimed at assessing the relative and combined effects of the bed roughness and shallowness on the time-averaged flow structure and the transverse fluctuating flow motion within a cylinder array (simulating rigid emergent vegetation). A particular attention was paid to: (i) the vertical distribution of time-averaged streamwise velocity and the helical secondary currents with a horizontal axis in the cylinder-wake ; (ii) the upward flow forming immediately downstream of the cylinder; (iii) the fluctuating flow motion in the cylinder-wake. The secondary purpose of the study was to quantify the free-surface waves that are induced by the vortex shedding behind the cylinders (when the flow is laterally confined). As we found that waves were sensitive to the shallowness level and bed roughness, a preliminary study of the bed roughness and shallowness effects on the waves was conducted.

The flow structure behind emergent circular cylinders was investigated by Dupuis et al. ${ }^{5}$. The authors have observed that the vertical profile of time-averaged streamwise velocity behind a cylinder could be divided into two distinct regions: a (quasi-) Constant Velocity Region (denoted as CVR) in the upper part of the flow; and a near-bed region featuring a velocity-bulge, whose magnitude could reach $35 \%$ of the velocity measured in the upper flow. They also found that the elevation of the local peak velocity was independent of flow rate, and the height of the velocitybulge region was independent of both flow rate and bed-surface state (smooth or rough). In the experiments of Liu et al. $^{3}$, the near-bed velocity-bulge was observed for both submerged and emergent rigid stems, and was combined with an upward flow located immediately downstream of the stems. A velocity-bulge was also identified in a model of double-layer vegetation (array of 
cylinders of two different heights) by Liu et al. ${ }^{11}$, behind the short (submerged) and tall (emerged) cylinders, and also behind the tall cylinders above the short cylinders. In the latter case, the upper and lower velocity-bulges could arise from a same physical phenomenon, the array of short cylinders acting as a bottom roughness on the upper flow.

Comparing the data of Liu et al. ${ }^{3}$ to Large-Eddy Simulations (LESs), Stoesser et al. ${ }^{12}$, Stoesser, Kim, and Diplas ${ }^{13}$ and Kim et al. ${ }^{2}$ found that the near-bed velocity-bulge and the upward flow were related to the presence of helical secondary flows with a longitudinal axis, which exist even with a moveable bed ${ }^{2}$. A pair of counter-rotating vortices develops behind each cylinder in the near-bed region. These vortices bring high-momentum fluid from the fast flow region into the wake with an upward fluid motion in the alignment of the cylinder. The vortices magnitude fastly decreases when moving downstream. The numerical results of Stoesser, Kim, and Diplas ${ }^{13}$ also indicate that the secondary flows vanish with a high vegetation density.

The origin of these secondary flows, also termed 'base vortices', was investigated by Sumner, Heseltine, and Dansereau ${ }^{14}$ in the case of a single circular cylinder of finite length. They suggested that they could be a time-averaged print of the von Karman vortices, which are actually bent near the bed due to the vertical gradient of mean steamwise velocity induced by the ground plane. This assumption has been confirmed by Wang and Zhou ${ }^{15}$, who experimentally studied the flow structure behind a single square cylinder of finite length. First, the cylinder-near-wake was found to be composed of three types of vortices: the well-known von Karman (or spanwise) vortices with a vertical axis, which were periodically shed in the wake; and also base and tip vortices with an horizontal axis that formed respectively near the ground plane and at the cylinder free end. Second, relying on two-point velocity measurements and space correlations of the velocity fluctuations, the authors have shown that tip, spanwise and base vortices were part of a single vortical structure. Last, they proposed a model of flow structure, in which both base and tip vortices were depicted as projections of the spanwise vortices curved under the effect of the mean velocity gradients induced by the ground plane and the cylinder tip.

The cylinder-near-wake structure was also found to be dependent on the vertical boundary layer thickness $\delta$ of the approaching flow. Wang et al. ${ }^{16}$ have investigated the wake of a square cylinder for various $\delta$-values. They have observed that, as $\delta$ increased, the upward flow became stronger and the length of the reversed flow region near the bed decreased. Similar results were obtained by Hosseini, Bourgeois, and Martinuzzi ${ }^{17}$ and El Hassan, Bourgeois, and Martinuzzi ${ }^{18}$ for circular cylinders of aspect ratios $A R=8$ and 4 , respectively. The cylinder aspect ratio is 
defined as $A R=h / \phi$, where $h$ is the cylinder height (equal to the time-averaged water depth $\bar{D}$ for emergent cylinders) and $\phi$ is the cylinder diameter (or its width in the case of square cylinders). These results are consistent with the wake structure model proposed by Wang and Zhou ${ }^{15}$ as a larger approaching boundary layer will result in more bent spanwise vortices.

For a single submerged cylinder, the structure of the wake is also influenced by the cylinder aspect ratio. For instance, Sumner, Heseltine, and Dansereau ${ }^{14}$ and Wang and Zhou ${ }^{15}$ have observed that the magnitude of the vertical flows, induced by base and tip vortices, was increasing with the cylinder aspect ratio. Compared to emergent cylinders, the wake structure is however strongly modified by the flow over the cylinder and its reattachment to the bed downstream of the cylinder. For submerged rigid canopies, the flow structure intra-canopy is modified by the vertical mixing layer (inflection point in the mean velocity profile at the canopy tip as shown by Chagot, Moulin, and Eiff ${ }^{19}$ ) and the associated Kelvin-Helmholtz vortices. The flow in the canopy is also modified by outer streamwise vortices that are generated by the external logarithmic layer and penetrate the canopy as recently shown by the LESs of Monti, Omidyeganeh, and Pinelli ${ }^{20}$.

Wang and Zhou ${ }^{15}$ also noticed that several studies do not report base vortices or their vanishing for small aspect ratios ${ }^{14,21-23}$. First, the absence of base vortices could be explained by a too small effect of the boundary layer. Second, since the base vortices fastly decay behind the cylinder, they could be missed if no measurements are made very close to the cylinder. This would be supported by the LESs of the flow past a submerged circular cylinder by Krajnovic ${ }^{24}$. With a relatively thin approaching boundary layer $(\delta / \phi=0.07)$ and an aspect ratio $A R=6$, the numerical results have confirmed the presence of small base vortices in the near-wake, which disappear quickly after the impingement of the downward flow on the ground plane.

The effect of the bed-surface state on the flow structure behind rigid vegetation was studied by several authors, but contradictory results were obtained. For instance, Dupuis et al. ${ }^{5}$ experimentally observed a larger magnitude of the velocity-bulge for a smooth bed than for a rough bed, while Liu et al. ${ }^{3}$ observed an opposite result. On the other hand, Martino, Paterson, and Piva ${ }^{4}$ do not report a near-bed velocity-bulge above a rough bed made of a layer of uniform glass-beads, relying on detailed PIV measurements, for both submerged and emerged cylinders. Investigating the wake behind a submerged to emergent single circular cylinder (of relatively small aspect ratios, $A R<2$ ) set on a smooth bed or rough bed, Sadeque, Rajaratnam, and Loewen ${ }^{25}$ found that, for the weakly submerged case, the bed roughness was responsible for an increase in the upward flow behind the cylinder. For the emergent case, a larger reversed flow zone was observed with the 
rough bed than with the smooth bed. These authors also noticed that, for both weakly submerged and emergent cases, the reverse flow zone was almost uniform on the whole cylinder height with the rough bed, which they ascribed to a bed stabilization effect on the cylinder wake. The bed stabilization effect has been described by Chen and Jirka $^{26}$ for cylinders of small aspect ratio. They observed that small flow depths and bed friction can stabilize the wake and suppress the vortex shedding. Using the shallow wake parameter $S=C_{f} \phi / \bar{D}$ introduced by Ingram and $\mathrm{Chu}^{27}, C_{f}$ being the bed friction coefficient, they defined three wake regimes: the vortex shedding regime occurring for small $S$-values $(S<0.2)$, the unsteady bubble regime occurring for intermediate $S$ values $(0.2<S<0.5)$ and the steady bubble regime occurring for large $S$-values $(S>0.5)$.

Based on the previous results of the literature, sometimes contradictory, we wanted to clarify the effects of the bed-surface state on the flow structure behind rigid emergent vegetation, and particularly, on the formation of the near-bed velocity-bulge and helical secondary currents, and also on the transverse velocity fluctuations in the cylinder wake. For that purpose, time-averageddepth-uniform flows through a cylinder array have been investigated in a laboratory open-channel flume with a smooth bed (glass bottom) and a rough bed (bottom covered by dense artificial grass). For each bed-surface state, we studied two shallowness levels (using two flow rates 7 and $131 \mathrm{~s}^{-1}$ ) to also assess the shallowness effects on the flow structure. In addition, we have conducted transient flow simulations using the code OpenFOAM to test the influence of a wide range of bed roughness heights on the flow structure (a task that is not simple in the laboratory), for the two different shallowness levels. Note that we have considered a fixed free surface (rigid lid assumption), which enabled to restrain the calculation domain to an elementary pattern of cylinders and to use cyclic boundary conditions.

As laterally confined flows through cylinder arrays can involve transverse free-surface oscillations (termed 'seiche waves') caused by the vortex shedding behind the cylinders ${ }^{5,28-31}$, the effects of the bed roughness and shallowness on the seiche waves were also experimentally explored. We wanted to confirm and extend previous results obtained by Dupuis et al. ${ }^{5}$ in the same flume (comparing two flow cases over a rough bed with different shallowness levels, they have observed seiche waves for the deeper flow, but only random oscillations for the shallower flow).

Section II below outlines the open-channel flume used in the experiments, the flow conditions and the techniques for measuring water levels and velocities. Section III presents the numerical method that was used to further investigate the effects of bed roughness and shallowness on the flow structure. Section IV provides information on the measured transverse and longitudinal mo- 
tions of water surface. The effects of the bed-surface state (smooth or rough) and shallowness on the seiching phenomenon are analyzed. Section V is dedicated to the study of the velocity fluctuations, relying on both the measurements and simulations of turbulence statistics. In particular, the lock-in process between vortex shedding and transverse surface waves is analyzed. Section VI presents the time-averaged flow structure. The effects of bed roughness and shallowness on the streamwise velocity-bulge and upward flow in the cylinder wake are assessed, based on both the experimental data and the numerical simulations (using a wide range of roughness heights). Finally, the main conclusions are drawn in section VII.

\section{EXPERIMENTS}

\section{A. Flume and flow conditions}

Experiments were performed in an $18 \mathrm{~m}$ long and $1 \mathrm{~m}$ wide open-channel flume (sketched in Fig. 1) at the Hydraulics and Hydro-morphology Laboratory of INRAE (HHLab), LyonVilleurbanne, France. The flume bed slope in the longitudinal direction, $S_{0}$, is $1.05 \times 10^{-3}$. Two different bed-surface states were used: a hydraulically-smooth bed (glass bottom); and a hydraulically-rough bed (bottom covered by dense artificial grass consisting of $1 \mathrm{~mm}$ wide rigid blades with a height $\varepsilon=5 \mathrm{~mm}$, the density being 256 blades per square centimeter). A staggered array of emergent circular cylinders was then placed over the smooth or rough bed along the whole flume working length, with a density of 81 cylinders per square meter $(9 \times 9)$. Each wooden cylinder has a diameter $\phi$ of $10 \mathrm{~mm}$, and the spacing between two contiguous cylinder rows, $L$, is $80 \mathrm{~mm}$. These dimensions (and also the hydraulic parameters described below) were chosen within the research project Flowres (https://flowres.inrae.fr/en/), framework of the present study. The cylinder array simulates a woodland at a 1:100 scale (see Dupuis et al. ${ }^{5}$ for further details).

Flow structure was studied within an elementary pattern of four cylinders that is sketched in Fig. 2, and whose position within the cylinder array is indicated in Fig. 1 by a rectangle with dotted-line sides. Two different Cartesian right-handed coordinate systems are used in the present study. The first one is linked to the flume (Fig. 1). The $x_{0^{-}}, y_{0^{-}}$, and $z_{0}$-axes are aligned with the longitudinal (along the flume), lateral, and vertical (normal to the flume bottom) directions, respectively. The system origin is defined as: $x_{0}=0$ at the flume entrance (outlet of the inlet 


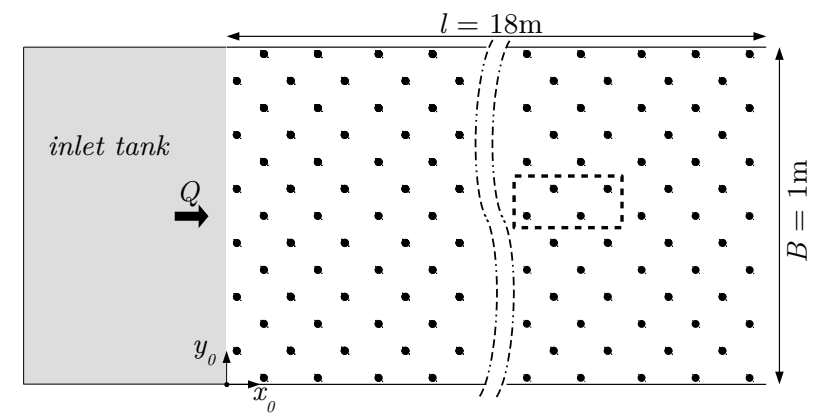

FIG. 1. Sketch of the open-channel flume (top view) with a staggered distribution of circular cylinders. The elementary cylinder pattern, in which velocity measurements were carried out, is bounded by a rectangle with dotted-line sides. The flow rate, the working length and width of the flume are denoted as $Q, l$ and $B$, respectively.

tank); $y_{0}=0$ at the right-hand side-wall of the flume; $z_{0}=0$ at the flume bottom (glass bottom or average level of the rigid blade tips). The second coordinate system, $\{x, y, z\}$, is linked to the elementary pattern, as depicted in Fig. 2.

Four streamwise uniform flows were studied, defined by a fairly constant time-averaged flow depth $\bar{D}$ in the longitudinal direction. For each type of bed-surface state (rough or smooth), two values of flow rate were considered, $Q=7$ and $131 \mathrm{~s}^{-1}$. The four flow cases are termed Q7s, Q7r, Q13s, and Q13r, where $r$ and $s$ refer to rough bed and smooth bed, respectively. For each case, streamwise time-averaged-depth-uniformity was achieved by adjusting the height of the downstream tail weir to get constant $\bar{D}$-values along $x$-axis. It should be noticed at this stage that, owing to the wakes superposition along the channel, the flow depth fluctuations will increase along the flume (to be seen in section IV). This means that flow resistance is not uniform in the longitudinal direction and uniform flow conditions cannot be exactly attained.

The flow parameters are given in table I, in which $U_{Q}=Q /(B \bar{D})$ is bulk velocity, $\operatorname{Re}_{\bar{D}}=\left(U_{Q} \bar{D}\right) / v$ is the Reynolds number based on water depth, $\mathrm{Fr}=U_{Q} / \sqrt{g \bar{D}}$ is the Froude number, $\lambda_{f}=(\bar{D} \phi) /\left(2 L^{2}\right)$ is the vegetation frontal density per unit ground area, $v$ is water kinematic viscosity, and $g$ is the acceleration due to gravity.

\section{B. Water level and velocity measurements}

Free-surface elevations were measured using an ultrasonic sensor (Baumer UNDK20I6912) with a resolution between 0.1 to $0.3 \mathrm{~mm}$. The acquisition rate was $50 \mathrm{~Hz}$ and the acquisition 


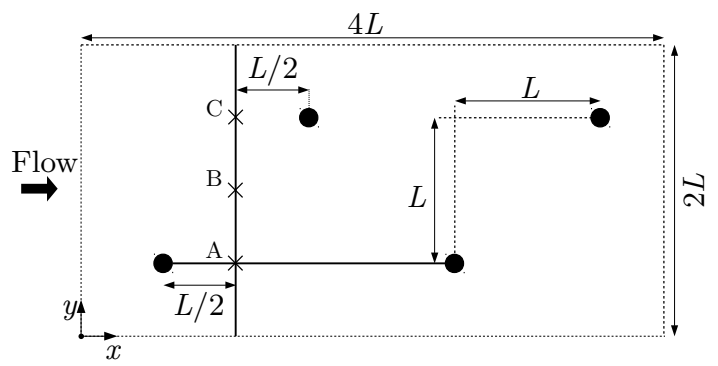

(a) Top view

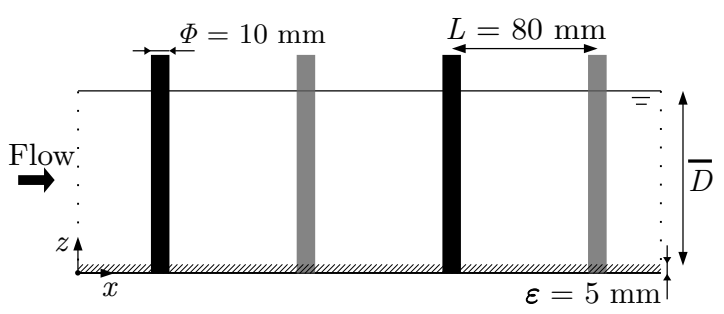

(b) Side view

FIG. 2. Sketch of the elementary pattern of cylinders seen from above $(a)$ and from the side $(b)$, with the locations of the longitudinal and transverse measuring planes (solid lines in the top view) and of the specific measuring points $\mathrm{A}, \mathrm{B}$ and $\mathrm{C}$. The parameters $\phi, L, \bar{D}$ and $\varepsilon$ refer to cylinder diameter, the spacing between two rows of cylinders, time-averaged water depth, and the average height of the artificial grass rigid blades, respectively.

TABLE I. Measured parameters of the flow cases: $Q$ is flow rate, $\bar{D}$ is time-averaged water depth, $U_{Q}=Q /(B \bar{D})$ is bulk velocity, $\operatorname{Re}_{\bar{D}}=\left(U_{Q} \bar{D}\right) / v$ is the flow depth Reynolds number, $\mathrm{Fr}=U_{Q} / \sqrt{g \bar{D}}$ is the Froude number, $\lambda_{f}=(\bar{D} \phi) /\left(2 L^{2}\right)$ is the frontal density per unit ground area.

\begin{tabular}{lccccccc}
\hline \hline Case & $\begin{array}{c}\text { Bed-surface } \\
\text { state }\end{array}$ & $\begin{array}{c}Q \\
\left(\mathrm{ls}^{-1}\right)\end{array}$ & $\begin{array}{c}\bar{D} \\
(\mathrm{~mm})\end{array}$ & $\begin{array}{c}U_{Q} \\
\left(\mathrm{~cm} \mathrm{~s}^{-1}\right)\end{array}$ & $\begin{array}{c}\mathrm{Re}_{\bar{D}} \\
(-)\end{array}$ & $\begin{array}{c}\text { Fr } \\
(-)\end{array}$ & $\begin{array}{c}\lambda_{f} \\
(-)\end{array}$ \\
\hline Q7s & smooth & 7 & 56 & 12.5 & 7000 & 0.17 & 0.044 \\
Q7r & rough & 7 & 56 & 12.5 & 7000 & 0.17 & 0.044 \\
Q13s & smooth & 13 & 95 & 13.7 & 13000 & 0.14 & 0.074 \\
Q13r & rough & 13 & 99 & 13.1 & 13000 & 0.13 & 0.077 \\
\hline \hline
\end{tabular}


duration at each measuring point was $300 \mathrm{~s}$. The latter ensures time convergence of both the timeaveraged flow depth $\bar{D}$ and of the standard deviation $\sigma_{D^{\prime}}$ of the water depth fluctuation $D^{\prime}$ (with $D^{\prime}(t)=D(t)-\bar{D}$, where $D(t)$ is the instantaneous water depth at time $t$ ).

First, time-averaged flow depth $\bar{D}$ was measured along the channel center line (at $y_{0} / B=0.5$ ) for each flow case. Denoting $\langle\bar{D}\rangle_{x}$ as the longitudinally- and time-averaged flow depth, the variations in $\bar{D}$ are of $\pm 0.5 \mathrm{~mm}$ around $\langle\bar{D}\rangle_{x}$, confirming the depth-uniformity of the test cases. Second, time series of the instantaneous flow depth $D(t)$ were recorded across the channel from $y_{0} / B=0.25$ to 0.75 at eight $x_{0}$-positions (eight transverse transects in total), to investigate the transverse oscillations of water surface. These measurements were taken at a spatial interval of $1 \mathrm{~cm}$ in the lateral direction, and were used to determine the standard deviation of flow depth fluctuation $\sigma_{D^{\prime}}$, the amplitude of surface oscillations $A$, and the predominant frequency of the surface wave $f_{s w}$ arising from the spectrum of the flow depth fluctuations. Third, for case Q13r, two ultrasonic sensors were simultaneously used to capture the longitudinal motion of the seiche waves. The two sensors were aligned with the $x_{0}$-axis at $y_{0} / B=0.5$, and time-series of $D(t)$ were recorded varying the longitudinal spacing $\Delta x$ between the two sensors. The time lag between the two signals, $\Delta t$, was determined by computing the cross-correlation function of the two signals.

The three instantaneous velocity components $u_{x}, u_{y}$ and $u_{z}$ were measured using an Acoustic Doppler Velocimeter (ADV) with a side-looking probe (Nortek Vectrino+) at a sampling rate of $100 \mathrm{~Hz}$. The flow was seeded with $55 \mu \mathrm{m}$ polyamid particles (Evonik Vestosint 1164) to improve acoustic signal quality, and to get typical signal-to-noise ratios higher than $20 \mathrm{~dB}$, as recommended by McLelland and Nicholas ${ }^{32}$. The ADV raw data were filtered using the despiking technique of Goring and Nikora ${ }^{33}$. The time convergence of the time-averaged velocity and turbulence statistics (at various positions within the elementary cylinder pattern) was obtained with an acquisition duration of 300 s. According to Proust and Nikora ${ }^{34}$, who carried out ADV measurements within an other type of shallow transversely sheared flows in the same flume, and with the same sampling rate and acquisition duration, the sampling standard errors for the key flow parameters were found to be approximately: $1 \%, 9 \%$ and $16 \%$ for the time-averaged velocities, $\overline{u_{x}}, \overline{u_{y}}$ and $\overline{u_{z}}$, respectively; $3 \%, 2 \%$ and $3 \%$ for the turbulence intensities $\sqrt{\overline{u_{x}^{\prime 2}}}, \sqrt{\overline{u_{y}^{\prime 2}}}$ and $\sqrt{\overline{u_{z}^{\prime 2}}}$, respectively; and $10 \%$ for the transverse Reynolds shear stress $-\overline{u_{x}^{\prime} u_{y}^{\prime}}$.

The flow structure of the four test cases was investigated along two vertical planes (Fig. 2): a $y-z$ plane passing between two lateral rows of cylinders and a $x-z$ plane aligned with a longitudinal cylinder row. Measurements were taken at a spatial interval of $5 \mathrm{~mm}$ in the two directions of each 
plane. Due to the ADV probe size, the first measurement points were located at $7 \mathrm{~mm}$ above the channel bottom, and the vertical velocity component $u_{z}$ could not be measured when the distance to the free surface was lower than $20 \mathrm{~mm}$. Note that, along the transverse plane $(y-z)$ located at $x / \phi=8$, the vertical distributions of the flow parameters have been more specifically studied at three measuring points: at point $\mathrm{A}$, located $4 \mathrm{~cm}$ downstream of a cylinder and at $y / \phi=4$; at point $\mathrm{B}$, in the free-stream region at $y / \phi=8$; and at point $\mathrm{C}, 12 \mathrm{~cm}$ downstream of a cylinder and at $y / \phi=12$.

It should be noted that a small misalignment of the ADV probe with the streamwise direction can lead to significant errors on the transverse velocity $u_{y}$ and vertical velocity $u_{z}$, as these two components are often small compared to the streamwise velocity $u_{x}$. The data of $u_{y}$ and $u_{z}$ were therefore corrected based on the formulas of Peltier et al. ${ }^{35}$. First, based on symmetry considerations within the cylinder array, we assumed that the spatial average of the time-averaged transverse velocity $\overline{u_{y}}$ in the transverse measuring plane (Fig. 2) was equal to zero. This resulted in a rotation around the vertical axis of a yaw angle $\theta_{z}$ lower than $3^{\circ}$. Second, we also assumed that the spatial average of the time-averaged vertical velocity $\overline{u_{z}}$ in the transverse measuring plane was equal to zero, resulting in a rotation around the transverse axis of a pitch angle $\theta_{y}$ lower than $2^{\circ}$. This hypothesis can be open to criticism as it is relevant at a local scale for a streamwise uniform velocity field without obstacles, but not in the case of a flow through a cylinder array. In the latter case, flow is uniform only at a macroscopic scale. In addition, vertical velocity $u_{z}$ could not be measured in the near-surface layer. Unfortunately, a more straightforward and relevant angular correction procedure for $u_{z}$ has not been found here. As in the numerical simulations (to be considered in section VI), the spatial average of $\overline{u_{z}}$ was found to be zero in the transverse measuring plane, we eventually relied on this hypothesis. However, we should keep in mind this assumption when comparing the numerical and measured values of the vertical velocity $\overline{u_{z}}$.

\section{NUMERICAL METHOD}

\section{A. Scope and objective of the simulations}

Transient simulations of the flows presented in section II have been performed to better understand the effect of the bed roughness on the vertical distribution of mean streamwise velocity and on the helical secondary flows forming downstream of the cylinders near the bed (previously 
termed 'bases vortices'). Based on a rigid lid assumption at the water surface, the simulations do not take into account the effect of the free-surface oscillations on the mean flow structure and fluctuating flow motion. Neglecting the surface oscillations enabled to model an elementary pattern of cylinders only. This assumption can be justified a priori for the modeling of the near-bed mean flow patterns, namely the flow acceleration and the associated helical secondary currents, as they are located in the lower part of the water column, a part that should be controlled by bed-friction effects. In contrast, this assumption is not strictly acceptable for the modeling of the transverse velocity fluctuations, as the gravity wave in the transverse direction is a pressure wave that can affect the fluctuating flow parameters. Therefore, we will have to be very careful when comparing either experimental and numerical turbulence statistics (to be analyzed in the next section V A), or experimental and numerical Strouhal numbers (next section V B). For instance, the numerical Strouhal numbers will not take into account the lock-in process between the seiche waves across the channel and the vortex shedding 28,29 .

Modeling the interplay between surface waves and flow structure would have required to model the channel width, i.e. multiple patterns of cylinders, using e.g. the Volume of Fluid method to simulate the water surface motion. In addition, modeling the effect of the longitudinal progressive wave would have required to model the whole flume length. Given the cylinder density $\left(81 \mathrm{~m}^{-2}\right)$, and given the cylinder diameter $(1 \mathrm{~cm})$ that imposes very fine mesh near the cylinder walls, the modeling of the flow over the whole width and length of the flume would result in a prohibitive number of cells ( 0.6 to 1.5 million cells being used for an elementary pattern of 4 cylinders).

\section{B. Simulations set-up}

The simulations were performed using the open-source CFD code OpenFOAM, which is based on the finite volume method. A hybrid RANS-LES turbulence model based on the Scale-Adaptive Simulation (SAS) approach has been used, namely the $k$ - $\omega$ SST-SAS model developed by Egorov

and Menter ${ }^{36}$. This model was previously used by $\mathrm{Yu}$, Zhan, and $\mathrm{Li}^{37}$ to simulate the flow through a vegetation patch.

With the $k-\omega$ SST-SAS model, the Navier-Stokes equations for an incompressible flow are solved in an unsteady RANS (URANS) framework, in which continuity and momentum conservation equations write:

$$
\frac{\partial \widetilde{u_{i}}}{\partial x_{i}}=0
$$




$$
\frac{\partial \widetilde{u_{i}}}{\partial t}+\widetilde{u_{j}} \frac{\partial \widetilde{u_{i}}}{\partial x_{j}}=-\frac{1}{\rho} \frac{\partial \widetilde{p}}{\partial x_{i}}+\frac{\partial}{\partial x_{j}}\left[v \frac{\partial \widetilde{u_{i}}}{\partial x_{j}}+\frac{R_{i j}}{\rho}\right],
$$

where $\widetilde{u_{i}}, \widetilde{p}, \rho$ and $v$ stand for the resolved (or filtered) instantaneous velocity component in the $i^{t h}$ direction, the resolved instantaneous pressure, water density and kinematic viscosity, respectively. The modeled Reynolds stresses, $R_{i j}$, are defined as follows:

$$
R_{i j}=\mu_{t}\left(\frac{\partial \widetilde{u_{i}}}{\partial x_{j}}+\frac{\partial \widetilde{u_{j}}}{\partial x_{i}}\right)-\frac{2}{3} \rho k \delta_{i j}
$$

where $\mu_{t}$ and $k$ stand for turbulent dynamic viscosity and turbulent kinetic energy, respectively, and $\delta_{i j}$ is the Kronecker symbol.

The $k-\omega$ SST-SAS model is derived from the well-known $k-\omega$ SST model $^{38}$ that solves the transport equations of turbulent kinetic energy $k$ and specific dissipation rate $\omega$. Readers can refer to Egorov and Menter ${ }^{36}$ for a complete description of the SAS model. These authors explain that, compared to the RANS $k-\omega$ SST model, an additional term in the transport equation of $\omega$ enables the SAS model to adjust the turbulence length-scale to the local flow inhomogeneity. This model is then designed to provide a RANS solution in stationary flow regions and to allow the break-up of large unsteady structures into a turbulent spectrum where flow features transient instabilities. The default values of the turbulence model coefficients were used in the simulations.

As the studied flows were depth-uniform in the streamwise direction, and as the width-todepth ratio $B / \bar{D}$ was sufficiently large to neglect side-walls effects, the computational domain was restricted to an elementary pattern of the cylinder array. Periodic conditions were applied along the streamwise and spanwise boundaries of the domain. A rigid lid assumption was adopted for the free-surface, with a free-slip condition. The gravity force was replaced by a longitudinal pressure gradient, which was iteratively computed to retrieve the actual bulk velocity $U_{Q}$. Noslip conditions were applied at each cylinder wall (similarly to the LESs of flows through rigid filamentous canopies by Monti, Omidyeganeh, and Pinelli ${ }^{20}$ ) and channel bottom. All the CFD boundary conditions depending on the flow cases studied are summarized in table II.

For the rough-bed flow cases, a classical wall function was used to model the bed roughness effect on the flow, following the approach used by Kim et al. ${ }^{2}$ for a similar flow configuration. The wall function used in OpenFOAM is a classical log law for the near-wall velocity:

$$
u^{+}=\frac{1}{\kappa} \ln \left(\frac{E y^{+}}{f_{\text {rough }}}\right),
$$


TABLE II. Boundary conditions in the simulations for cases Q7s, Q13s, Q7r and Q13r. The simulations presented in section IV are based on the same conditions, and the $k_{s}$ value was varied from 0.6 to $10 \mathrm{~mm}$

\begin{tabular}{lcc}
\hline \hline Flow case & Q7s and Q13s & Q7r and Q13r \\
\hline Bottom & no-slip condition & no-slip condition, rough-wall function \\
& $k_{s}=6$ and $7 \mathrm{~mm}$, respectively \\
\hline Cylinder wall & no-slip condition \\
\hline Water surface & rigid lid assumption and free-slip condition \\
\hline Inlet and outlet flow conditions & periodic \\
\hline Flow conditions at lateral boundaries & periodic \\
\hline
\end{tabular}

where $u^{+}=u / u_{\tau}$ and $y^{+}=y u_{\tau} / v$ are the normalized velocity and distance to the adjacent wall, respectively, $u_{\tau}=\sqrt{\tau / \rho}$ being the friction velocity, and $\tau$ the wall shear stress.

In Eq. (4), $\kappa=0.41$ is the von Karman constant, $E=9.8$ is an empirical constant, and $f_{\text {rough }}$ is a roughness function. The latter depends on the boundary layer regime. Introducing the normalized equivalent sand grain diameter $k_{s}^{+}=k_{s} u_{\tau} / v$, three different regimes can be defined: smooth boundary layers for $k_{s}^{+} \leq 2.25$; transitional regime for $2.25<k_{s}^{+}<90$; and rough boundary layers for $k_{s}^{+} \geq 90$. For these three regimes, the roughness function is respectively defined by:

$$
\begin{aligned}
k_{s}^{+} \leq 2.25: & f_{\text {rough }}=1, \\
2.25<k_{s}^{+}<90: & f_{\text {rough }}=\left[\frac{k_{s}^{+}-2.25}{87.75}+C_{s} k_{s}^{+}\right]^{\alpha}, \\
k_{s}^{+} \geq 90: & f_{\text {rough }}=1+C_{s} k_{s}^{+},
\end{aligned}
$$

where the exponent $\alpha$ writes:

$$
\alpha=\sin \left[0.4258\left(\ln \left(k_{s}^{+}\right)-0.811\right)\right] .
$$

The constant $C_{S}$ is usually defined as a roughness factor that can be calibrated depending on the roughness type. For a hydraulically-rough regime, the wall function (4) yields:

$$
u^{+}=\frac{1}{\kappa} \ln \left(\frac{E y^{+}}{1+C_{s} k_{s}^{+}}\right) \approx \frac{1}{\kappa} \ln \left(\frac{E y^{+}}{C_{s} k_{s}^{+}}\right) .
$$

The equivalent sand grain diameter $k_{s}$ of the dense artificial grass used in this study was previously estimated by Dupuis ${ }^{39}$. These authors fitted a similar logarithmic law to the profiles of 
measured time-averaged streamwise velocity for uniform flows over the same rough bed without cylinder array. The parameter $k_{s}$ was found to be equal to $6 \mathrm{~mm}$ and $7 \mathrm{~mm}$ for $Q=71 \mathrm{~s}^{-1}$ and $151 \mathrm{~s}^{-1}$, respectively.

In the first part of this study, $k_{s}$-values of $6 \mathrm{~mm}$ and $7 \mathrm{~mm}$ were imposed for the simulations of cases Q7r and Q13r, respectively. The constant $C_{s}$ was fixed at 0.3 to retrieve the law used by Dupuis ${ }^{39}$ for a hydraulically-rough regime. In the second part of this study, various $k_{s}$-values have been used to assess the influence of the bed-surface state on the flow structure. The tested $k_{s}$-values were in the range 0.6 to $10 \mathrm{~mm}$, corresponding to normalized sand grain diameter $k_{s}^{+}$ between 4.8 and 135.8 (from transitional- to fully-rough regimes).

As depicted in Fig. 3, the computational grid consisted in orthogonal cells (of $2.5 \mathrm{~mm}$ side) in the free-stream regions, and of hexahedral cells when approaching the cylinder walls. The size of the hexahedral cells decreases to resolve the viscous sublayer at cylinder walls. The height of the cell the closest to the cylinder wall is $0.05 \mathrm{~mm}$. The normalized wall distance at the first cell center, $y^{+}=y_{c} u_{\tau} / v$ ( $y_{c}$ being the normal distance from the first cell center to the adjacent wall), was found to be lower than 2. For the smooth-bed cases, the mesh was also refined near the bottom surface, leading to $y^{+}<5$ (i.e., a minimum height of the cells adjacent to the bottom of $0.64 \mathrm{~mm}$ ), excepted in the vicinity of cylinders where this value ranged from 5 to 10 . For the rough-bed cases, a fixed height of $5 \mathrm{~mm}$ was imposed to the first layer of cells adjacent to the bottom, to ensure the wall function validity with cells centers remaining in the log region of the boundary layer. A sensitivity analysis to the mesh refinement was performed for case Q7s. Very similar results were obtained for the regular grid and a finer grid that featured twice-smaller cells outside the boundary layer. This confirmed that spatial resolution was sufficient for the computation of the time-averaged flow and turbulence statistics.

Last, a second order Crank-Nicholson scheme was used for the temporal discretization and an adjustable time-step was applied to ensure that the maximum Courant numbers remained less than 1. A characteristic eddy turnover time ${ }^{13} t_{e}=\bar{D} / u_{\tau}$ can be defined based on the friction velocity estimated by $u_{\tau}=\sqrt{g S_{0} \bar{D}}$. The transient simulations were run over $100 t_{e}$ to ensure temporal convergence, and the computation of the flow statistics were started after $20 t_{e}$ to ensure that initial transients had no influence on the results.

The computations were performed using the cluster HIICS located at CINES, Montpellier, France. The cluster comprised 18 Nodes (432 cores, with $64 \mathrm{~Gb}$ of RAM for each core) with a node interconnection frequency of $40 \mathrm{~Gb} / \mathrm{s}$. The computations were parallelized on 144 cores 


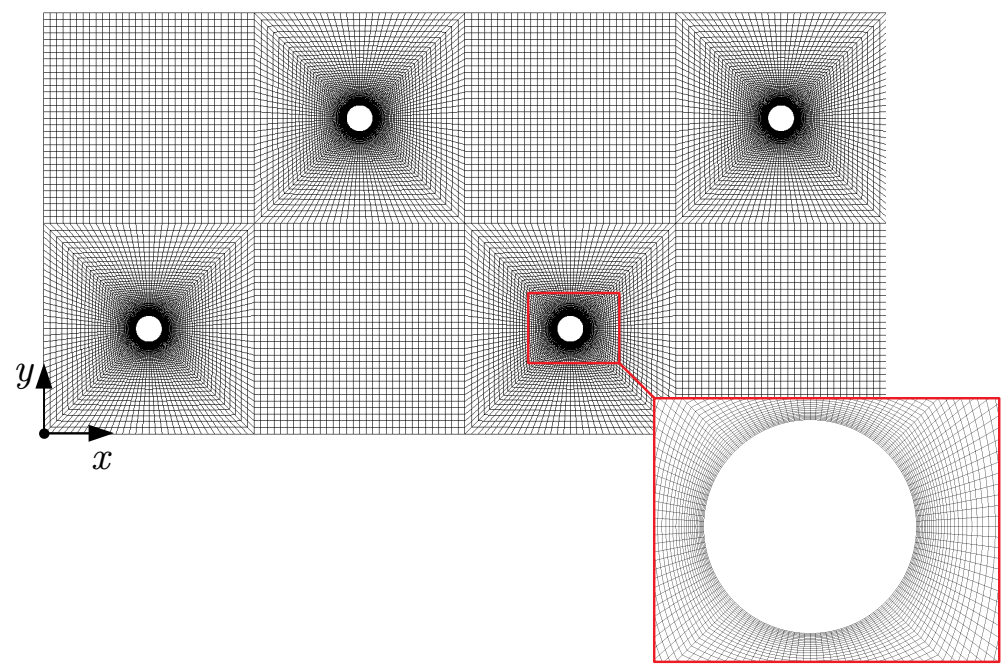

(a) Top view

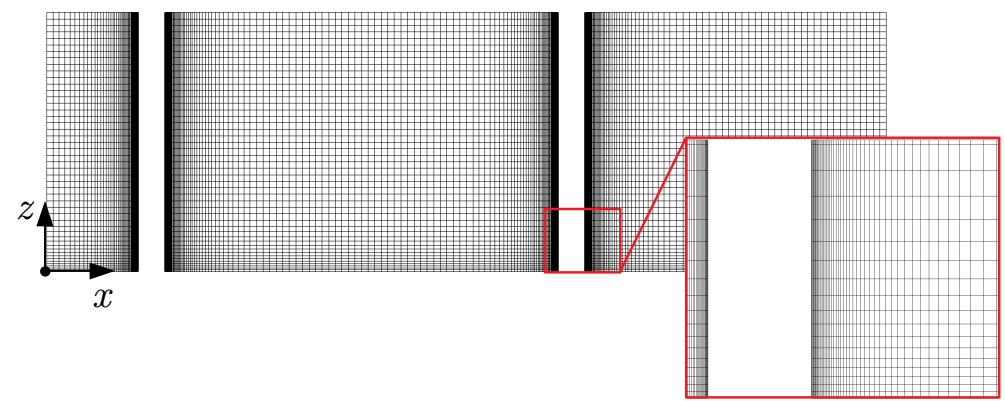

(b) Side view

FIG. 3. Computational grid used for the transient simulations of case Q13s, consisting in orthogonal cells with a constant size away from the cylinders, and in hexahedral cells of decreasing size when approaching the cylinder walls: $(a)$ top view with a zoom in the near-cylinder region; $(b)$ side view with a zoom in the near-bed region.

distributed on six nodes for the two cases with the higher flow rate, and on 96 cores distributed on four nodes for the two cases with the lower flow rate. The computational times ranged from $8 \mathrm{~h}$ (lower flow rate and rough bed, i.e. case Q7r) to $48 \mathrm{~h}$ (higher flow rate and smooth bed, i.e. case Q13s). 


\section{FREE-SURFACE OSCILLATIONS}

\section{A. General view}

Noticeable free-surface oscillations were measured for cases Q7s, Q13s and Q13r, with a maximum amplitude of the flow depth fluctuations $A_{D^{\prime}}^{\max }$ of the same order of magnitude as the cylinder diameter, and a maximum relative amplitude $A_{D^{\prime}}^{\max } / \bar{D}$ equals to $7.7 \%, 5.3 \%$ and $6.6 \%$, respectively (table III). These oscillations were visualized using a video camera placed at the flume outlet and looking upstream; they are shown in Fig. 4 (Multimedia view). Note that each video has been slowed down by dividing the frame rate by four to highlight both the transverse and longitudinal motions of the free-surface, namely a transverse standing wave and a longitudinal progressive wave moving upstream.

It is important to recall at this stage, that the transverse waves are produced by the periodic lift forces applying on the cylinders due to vortex shedding ${ }^{28}$. A necessary condition for the formation of these transverse waves, also termed seiche waves or seiching, is for the vortex shedding frequency to be close to a natural frequency of the transverse waves in open channel. In this case, Sarkar ${ }^{29}$ explains that a coupling mechanism, also termed 'lock-in', occurs. The vortex shedding for the different cylinders is in phase and the vortex shedding frequency becomes equal to one of the possible transverse waves frequencies. Such transverse waves have been observed for aligned $^{40}$, staggered ${ }^{5}$ and random distributions of cylinders ${ }^{29}$. In addition, the presence of longitudinal waves (combined with transverse waves) has also been reported in several studies ${ }^{28-31}$.

Interestingly, the free-surface oscillations are much weaker for case $\mathrm{Q} 7 \mathrm{r}\left(A_{D^{\prime}}^{\max } / \bar{D}=0.9 \%\right)$, reflecting that the seiche waves could be hindered or/and damped by the combined effects of a strong shallowness and a rough bed.

\section{B. Transverse oscillations}

The transverse standing waves can be highlighted by the transverse profiles of the standard deviation of flow depth fluctuations $\sigma_{D^{\prime}}$, which are shown in Fig. 5 for two $x_{0} / B$-positions. For cases Q7s, Q13s and Q13r, the $\sigma_{D^{\prime}}$-profiles feature noticeable periodic oscillations, whose amplitude increases when moving downstream. Only the modes 6 and 7 are excited, as observed by Dupuis et al. ${ }^{5}$ for flows with discharges of 7,15 , and $21 \mathrm{ls}^{-1}$. The mode 7 is observed for case Q7s all along the flume, with a node at the channel center line $\left(y_{0} / B=0.5\right)$, and a transverse wavelength 
TABLE III. Features of the free-surface oscillations: measured maximum amplitude $A_{D^{\prime}}^{\max }$ and standard deviation $\sigma_{D^{\prime}}^{\max }$ of the flow depth fluctuations (maximum values of the measurements taken at height various $x_{0}$-positions); measured frequency $f_{D^{\prime}}$ (average value over the height $x_{0}$-positions) associated with oscillation mode $n=6$ and 7; theoretical frequency $f_{D^{\prime}}^{\text {th }}$ derived from Eq. (10) for $n=6$ and 7; and predominant mode observed along the transverse profiles of $\sigma_{D^{\prime}}$.

\begin{tabular}{|c|c|c|c|c|c|c|c|}
\hline Case & $\begin{array}{l}A_{D^{\prime}}^{\max } \\
(\mathrm{mm})\end{array}$ & $\begin{array}{l}\sigma_{D^{\prime}}^{\max } \\
(\mathrm{mm})\end{array}$ & $\begin{array}{c}f_{D^{\prime}}(n=6) \\
(\mathrm{Hz})\end{array}$ & $\begin{array}{c}f_{D^{\prime}}^{t h}(n=6) \\
(\mathrm{Hz})\end{array}$ & $\begin{array}{c}f_{D^{\prime}}(n=7) \\
(\mathrm{Hz})\end{array}$ & $\begin{array}{c}f_{D^{\prime}}^{t h}(n=7) \\
(\mathrm{Hz})\end{array}$ & $\begin{array}{c}\text { Predominant } \\
\text { mode }\end{array}$ \\
\hline Q7s & 4.3 & 3.0 & 1.90 & 1.92 & 2.10 & 2.15 & 7 \\
\hline Q7r & 0.9 & 0.8 & 1.96 & 1.92 & 2.14 & 2.15 & - \\
\hline Q13s & 5.0 & 3.5 & 2.07 & 2.10 & 2.25 & 2.30 & $6-7^{*}$ \\
\hline Q13r & 6.5 & 4.5 & 2.07 & 2.11 & 2.34 & 2.31 & 6 \\
\hline
\end{tabular}

* Longitudinal transition in mode from upstream to downstream

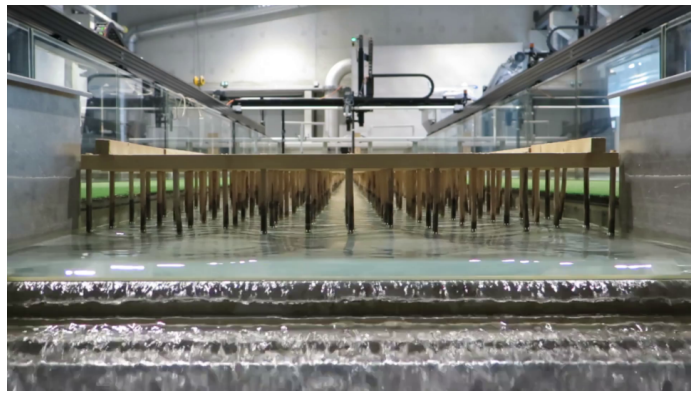

(a) Q7s

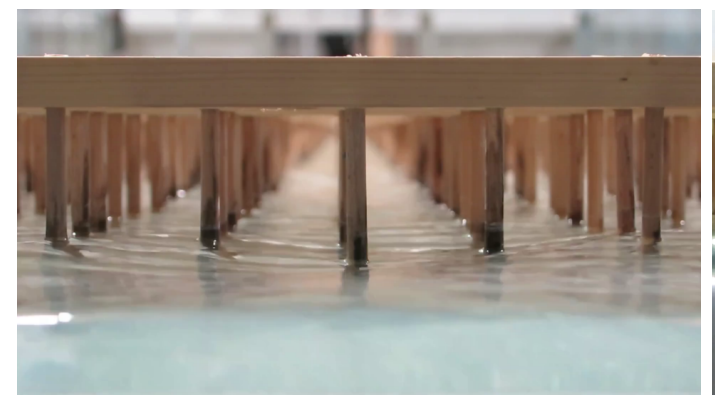

(c) Q13s

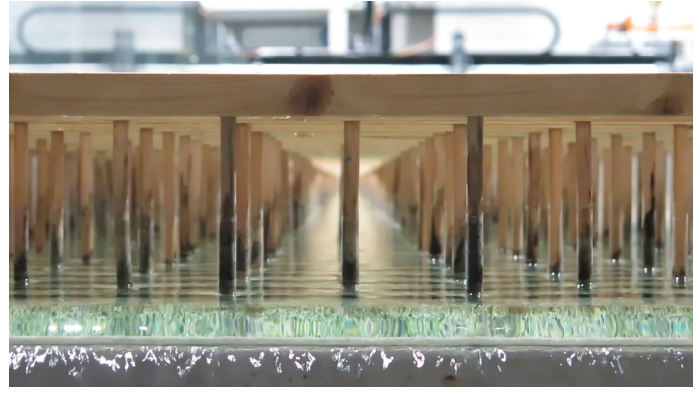

(b) Q7r

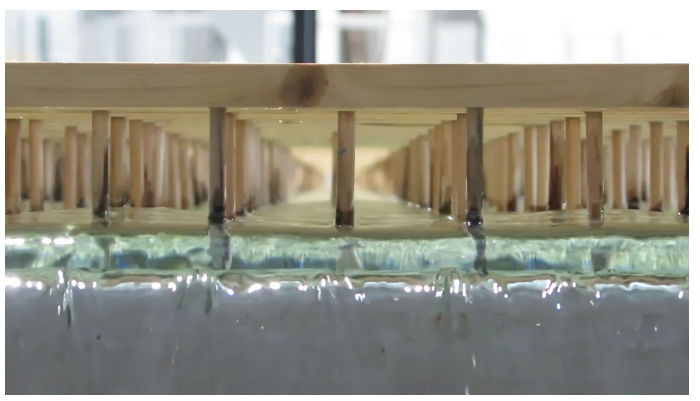

(d) Q13r

FIG. 4. Visualization of the free-surface oscillations using a video camera placed at the flume outlet and looking upstream (multimedia view) for flow cases (a) Q7s, (b) Q7r, (c) Q13s, and (d) Q13r. Each video has been slowed down by dividing the frame rate by four. 
$\gamma_{y}=280 \mathrm{~mm}$ (twice the distance between two adjacent anti-nodes). The mode 6 is observed for case Q13r, associated with $\gamma_{y}=330 \mathrm{~mm}$. Case Q13s is more intriguing, as it features a mode 6 at $x_{0} / B=4.2$ and a mode 7 at $x_{0} / B=12.8$, the transition in mode occurring at $x_{0} / B \approx 7$. Last, no oscillation mode is clearly visible for case Q7r, as the very small $\sigma_{D^{\prime}}$-values are of the same order of magnitude as the accuracy on water level measurement. The observed modes along the $\sigma_{D^{\prime}}$-profiles are reported in table III.

Fig. 5 also shows the transverse profiles of the main frequency arising from the spectrum of flow depth fluctuations, $f_{D^{\prime}}$. An interesting result is observed for the smooth-bed cases Q7s and Q13s (see Fig. 5a and Fig. 5c, respectively): the main frequency $f_{D^{\prime}}$ clearly differs at anti-nodes and nodes. To understand this result, we have compared the measured frequencies $f_{D^{\prime}}$ to the theoretical frequencies $f_{D^{\prime}}^{\text {th }}$ associated with small amplitude waves in open channels, which can be calculated using the following formula ${ }^{41}$ :

$$
f_{D^{\prime}}^{t h}(n)=\sqrt{\frac{g n}{4 \pi B} \tanh \left(\frac{n \pi \bar{D}}{B}\right)},
$$

The theoretical frequencies $f_{D^{\prime}}^{\text {th }}$ for the modes $n=6$ and 7 are also presented in Fig. 5 (dashed and dotted lines). For cases Q7s and Q13s, a very good agreement is observed between measured and theoretical frequencies. At anti-nodes, the main frequency is related to the mode observed along the $\sigma_{D^{\prime-}}$ profiles: $n=7$ for Q7s; $n=6$ at $x_{0} / B=4.2$ and $n=7$ at $x_{0} / B=12.8$ for Q13s. At nodes, on the other hand, the measured frequency $f_{D^{\prime}}$ is very close to $f_{D^{\prime}}^{\text {th }}(n=6)$ for Q7s; and for Q13s, $f_{D^{\prime}} \approx f_{D^{\prime}}^{t h}(n=7)$ at $x_{0} / B=4.2$ and $f_{D^{\prime}} \approx f_{D^{\prime}}^{t h}(n=6)$ at $x_{0} / B=12.8$. The results obtained for case Q13r do not contradict the previous ones: $f_{D^{\prime}}$ is fairly constant across the channel and close to $f_{D^{\prime}}^{\text {th }}(n=6)$, in agreement with the mode associated with the $\sigma_{D^{\prime}}$-profiles; and a higher frequency, close to $f_{D^{\prime}}^{\text {th }}(n=7)$, is captured at isolated points that are essentially nodes. Last, for case Q7r, though the $f_{D^{\prime}}$-data are more scattered, two main frequencies (close to $f_{D^{\prime}}^{t h}(n=6)$ and $f_{D^{\prime}}^{t h}(n=7)$ ) can be observed at $x_{0} / B=4.2$, and only one (close to $f_{D^{\prime}}^{t h}(n=7)$ ) at $x_{0} / B=12.8$.

Averaged frequencies for the modes 6 and 7 were derived from the local measurements of $f_{D^{\prime}}$ and reported in table III (denoted as $f_{D^{\prime}}(n=6)$ and $f_{D^{\prime}}(n=7)$ ), along with the theoretical values. To discriminate the measuring points featuring a main frequency related to mode 6 or 7 , a threshold value was defined by $\left(f_{D^{\prime}}^{\text {th }}(n=6)+f_{D^{\prime}}^{\text {th }}(n=7)\right) / 2$. The frequencies $f_{D^{\prime}}(n=6)$ and $f_{D^{\prime}}(n=7)$ were then computed as the average of the frequencies being respectively below and above this threshold value. As shown in table III, the averaged experimental frequencies are very close to the theoretical ones for all cases, with a relative discrepancy lower than $\pm 2.5 \%$. 

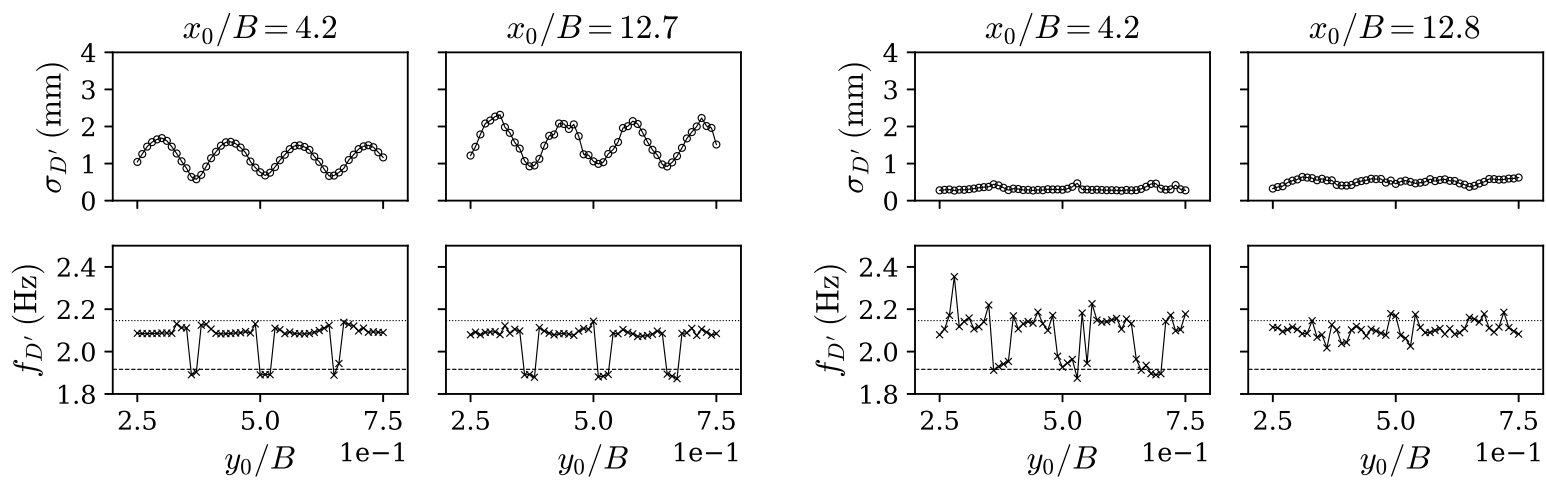

(a) Q7s

(b) Q7r
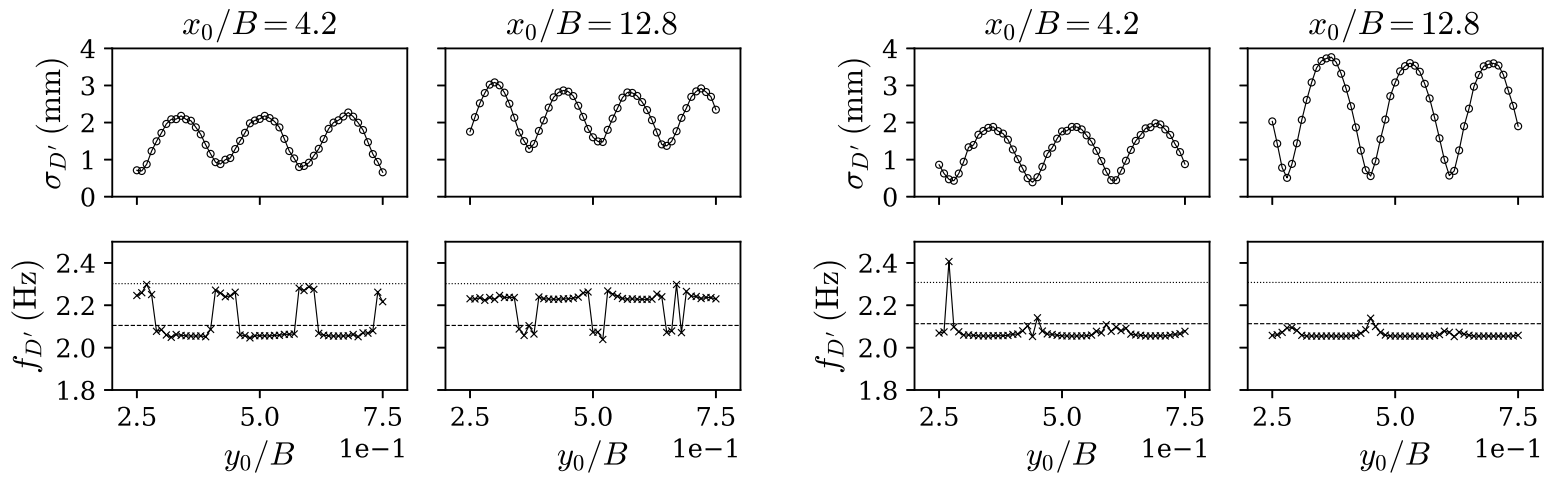

(c) Q13s

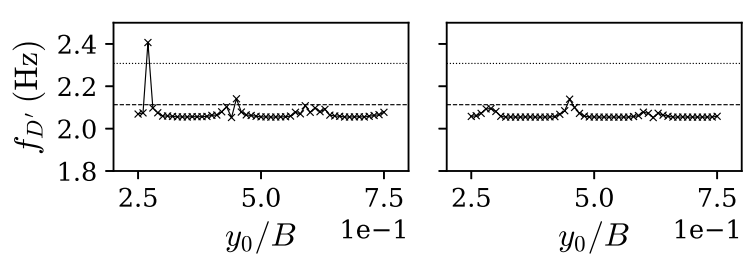

(d) Q13r

FIG. 5. Standard deviation of the flow depth fluctuation $\sigma_{D^{\prime}}$ and main frequency of the surface waves $f_{D^{\prime}}$ arising from the spectrum of the flow depth fluctuation $D^{\prime}$, as a function of lateral coordinate $y_{0} / B$, for flow cases (a) Q7s, (b) Q7r, (c) Q13s, and (d) Q13r. Measurements were taken at two longitudinal positions $x_{0} / B$. In the $f_{D^{\prime}}$-plots, the dotted and dashed lines indicate the theoretical frequency of the mode $n=7$ and $n=6$, respectively

The co-existence of two main frequencies or modes at a given $x_{0}$-position is actually visible in the spectra of flow depth fluctuations $D^{\prime}$. For instance, for case Q7s, Fig. 6 shows the spectrum of $D^{\prime}$ at $x_{0} / B=4.2$ for various $y_{0} / B$-positions ranging from $y_{0} / B=0.44$ (anti-node position) to $y_{0} / B=0.51$ (node position). At the anti-node, the spectrum features a peak at $2.09 \mathrm{~Hz}$ corresponding to the oscillation mode 7 (table III). At the node, the peak is observed at $1.89 \mathrm{~Hz}$, which corresponds to the mode 6 . Between the anti-node and the node, two peaks with the same magnitude can co-exist (at $y_{0} / B=0.49-0.50$ ). Since the peak magnitude is higher at the anti-node than at the node, we can thus conclude that there is a predominant mode (or frequency) that primarily 
drives the transverse distribution of $\sigma_{D^{\prime}}$, and also a secondary mode that is responsible for the non-zero oscillations measured at nodes. Note that the superposition of two modes is possible because high modes are activated and their frequencies are close to each other (discrepancy between $f_{D^{\prime}}(n=6)$ and $f_{D^{\prime}}(n=7)$ lower than $0.25 \mathrm{~Hz}$, i.e. a relative discrepancy of $\left.13 \%\right)$.

Lastly, it is important to notice that we have observed a great sensitivity of the free-surface oscillation amplitude to experimental conditions, as reported by Defina and Pradella ${ }^{30}$. For instance, small fluctuations of the flow rate $Q$ (less than $1 \%$ ) could change the predominant mode for Q7s (from 7 to 6), or modify the streamwise position of the longitudinal change in the predominant mode (from 6 to 7) for case Q13s. This longitudinal transition in mode could be caused by a slight misalignment of the cylinders, unavoidable along a $18 \mathrm{~m}$ long cylinder array with 81 cylinders per square meter. Note that a longitudinal change in mode was also observed in the same flume by Chetibi, Proust, and Benmamar ${ }^{40}$ for streamwise depth-non-uniform flows through cylinders with a square cross-section. However, in their experiments, the change in mode (from $n=2$ to 3 ) was due to noticeable changes in flow depth along the flume.

\section{Longitudinal oscillations}

The longitudinal motion of the free-surface is noticeable for cases Q7s, Q13s, and Q13r, as shown in the videos associated with Fig. 4. This motion was further investigated for case Q13r. Fig. 7 presents time-series of the flow depth fluctuations $D^{\prime}$, which were recorded using two ultrasonic probes aligned with the channel centerline (position of an anti-node as shown in Fig. 5d). The downstream probe is fixed at $x_{0}=12.75 \mathrm{~m}$, and the upstream probe is located at a variable distance $\Delta x$ from the downstream probe. Fig. 7 indicates that a longitudinal progressive wave goes back upstream, as observed by Defina and Pradella ${ }^{30}$ and Viero, Pradella, and Defina ${ }^{42}$.

The longitudinal and transverse waves, which have the same frequency, can be seen as two components of the same surface motion. Such a combination was termed metachronal wave by Defina and Pradella ${ }^{30}$. According to these authors, the longitudinal motion can be ascribed to a lack of synchronization of the vortex shedding behind cylinders. Another assumption can be put forward. The video for Q7s in Fig. 4 shows that, in the region without cylinders near the flume outlet, the longitudinal wave could be generated very close to the weir and moves upstream. As

mentioned by Defina and Pradella ${ }^{30}$, the longitudinal progressive wave could be attributed to a reflection of the standing wave. However, in the experiments of Viero, Pradella, and Defina ${ }^{42}$, the 


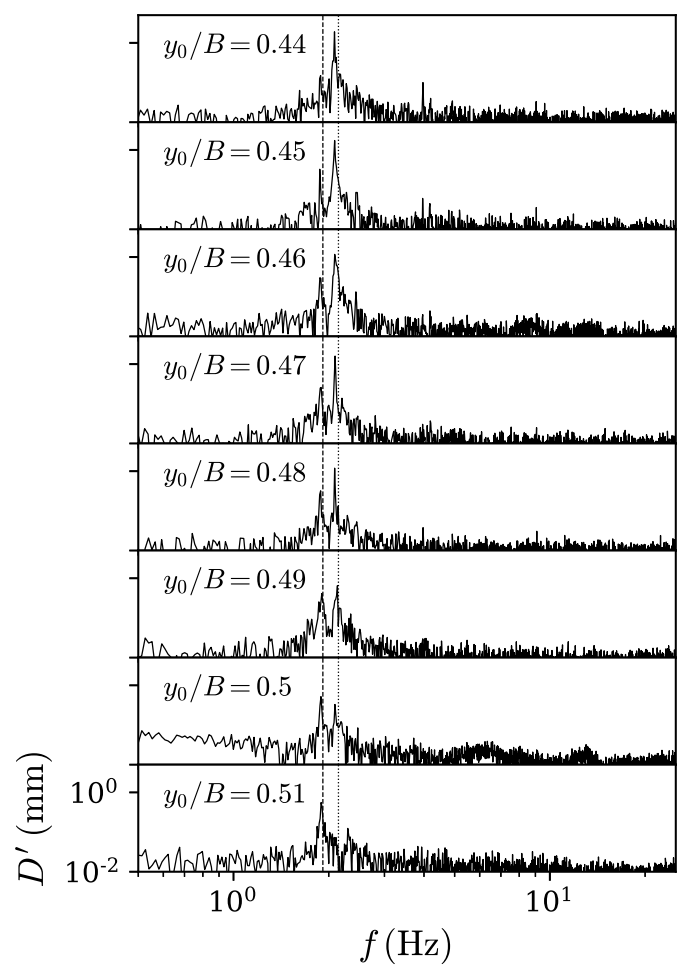

FIG. 6. Spectral decomposition of the flow depth fluctuations $D^{\prime}$ for case Q7s. Measurements at longitudinal position $x_{0} / B=4.2$, and at various lateral coordinates $y_{0} / B$ ranging from an anti-node position (0.44) to a node position (0.51). The frequencies $f_{D^{\prime}}^{t h}(n=6)$ and $f_{D^{\prime}}^{t h}(n=7)$ are indicated by dashed and dotted lines, respectively.

use of a vertical sluice gate (instead of a weir) leads to the same longitudinal component of the metachronal wave and to the same characteristics as that observed by Defina and Pradella ${ }^{30}$. It is therefore unlikely that the longitudinal wave is due to a reflection of the transverse wave.

A linear increase of the time lag $\Delta t$ between the upstream and the downstream signals with the distance between probes $\Delta x$ can be observed in Fig. 8. The longitudinal wave wavelength $\gamma_{x}$ and celerity $c_{x}$ have been estimated based on Fig. 8 using a linear regression. First, since the transverse and longitudinal waves have the same period ${ }^{42}$, when the time lag $\Delta t$ is equal to the half-period of the transverse wave $\left(1 / 2 T_{D^{\prime}}\right)$, the two signals feature opposite phases (roughly $\Delta x=640 \mathrm{~mm}$ in Fig. 7) and the distance between probes, $\Delta_{x}$, is equal to $1 / 2 \gamma_{x}$. The longitudinal wavelength was then estimated $\left(\gamma_{x}=1.246 \mathrm{~m}\right)$. Second, the longitudinal celerity $c_{x}$ was estimated from the slope of the linear regression, with $c_{x}=2.550 \mathrm{~m} \mathrm{~s}^{-1}$. Note that the transverse wave celerity $c_{y}$ (equal to $\left.\gamma_{y} f_{D^{\prime}}(n=6)\right)$ was found to be $0.683 \mathrm{~ms}^{-1}$. The progressive longitudinal wave has therefore 


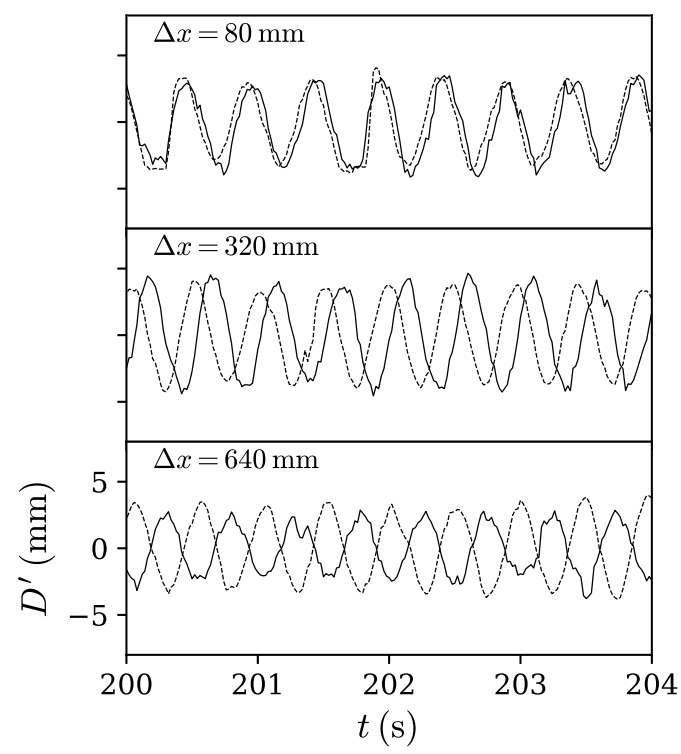

FIG. 7. Time-series of the flow depth fluctuations, $D^{\prime}$, for case Q13r, recorded using two ultrasonic probes aligned with the channel center line. The downstream probe (signal indicated by a dashed line) is fixed at $x_{0}=12.75 \mathrm{~m}$, and the upstream probe (signal indicated by a solid line) is at a variable distance $\Delta x$ from the downstream probe.

greater wavelength and celerity than the transverse wave, as observed by Viero, Pradella, and Defina $^{42}$.

\section{Effects of the shallowness and bed roughness}

The effects of the shallowness and bed-surface state on the free-surface waves can be seen in Fig. 9, where the maximal and minimal values across the channel of the standard deviation $\sigma_{D^{\prime}}$ (denoted as $\max \left(\sigma_{D^{\prime}}\right)$ and $\min \left(\sigma_{D^{\prime}}\right)$, respectively) are plotted as a function of the $x_{0}$-position. We recall that $\max \left(\sigma_{D^{\prime}}\right)$ and $\min \left(\sigma_{D^{\prime}}\right)$ are observed at anti-nodes and nodes, and are associated with the predominant and secondary modes, respectively. For a given bed-surface state, flow depth fluctuations decrease with an increasing shallowness. This is observed for both modes (Fig. 9a and Fig. 9b). For a given shallowness level, the second mode fluctuations are smaller for the rough-bed cases than for the smooth-bed cases (Fig. 9a). On the other hand, the predominant mode fluctuations are damped for the smaller discharge over rough bed only (Fig. 9b).

To understand these results, we will analyze the spectral signature of the transverse velocity 


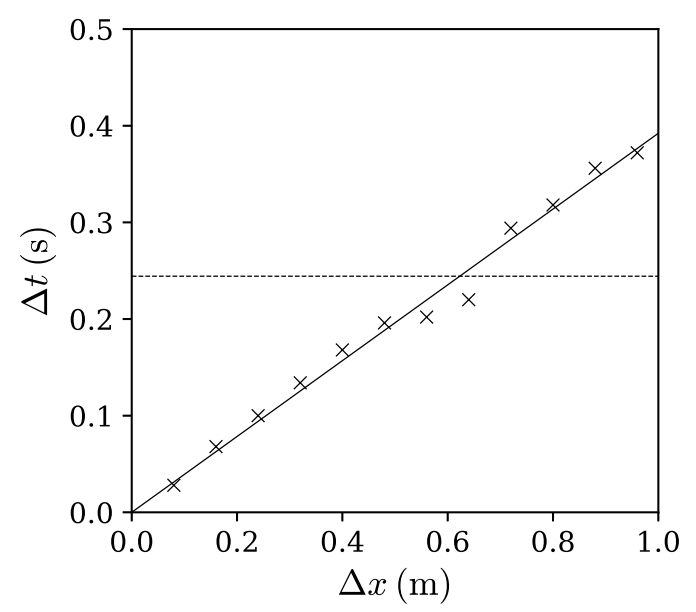

FIG. 8. Time lag $\Delta t$ between the two signals as a function of the longitudinal distance $\Delta x$ between the two probes, for case Q13r. The solid line represents the linear regression of the experimental data, and the dashed line represents the half-period of the transverse waves $T_{D^{\prime}} / 2=1 /\left(2 f_{D^{\prime}}\right)$ for $n=6$, see table III.

fluctuations over the water column, since surface oscillations and transverse velocity fluctuations are linked by the lock-in process between seiche waves and vortex shedding. Power density spectra $S_{y y}$ of the transverse velocity fluctuation $u_{y}^{\prime}$ behind a cylinder at point A (see Fig. 2a) and in the free-stream region at point B are shown in Fig. 10. For each point, measurements were taken near the bed at $z / \phi=1$, and higher in the water column at $z / \phi=2.5$ for cases Q7s,r and at $z / \phi=4$ for Q13s,r. Note that velocity fluctuations will be further analyzed in the next section.

First, the spectra of velocity fluctuation $u_{y}^{\prime}$ highlight the lock-in process, as the peaks in $S_{y y}$ correspond to the main frequencies of the seiche waves (compare Fig. 10 to Fig. 5). For instance, the spectra for the smooth-bed cases behind a cylinder at point A (solid black lines) feature a noticeable peak associated with vortex shedding for a frequency around $2 \mathrm{~Hz}$, which is the predominant frequency of the surface oscillations $(2.10 \mathrm{~Hz}$ and $2.25 \mathrm{~Hz}$ for Q7s and Q13s, respectively, see table III).

Second, the spectra of velocity fluctuation $u_{y}^{\prime}$ highlight the bed-surface state effect. At point A near the bed $(z / \phi=1)$, the magnitude of the peak in $S_{y y}$ is lower for the rough-bed cases than for the smooth-bed cases (Figs. 10a-b and Figs. 10e-f, solid black lines), e.g. by one decade for 13 $1 \mathrm{~s}^{-1}$. This would suggest that, near the bed, the horizontal fluctuating motion is disorganized, hindered, by a higher 3D bed-induced turbulence over the rough bed than over the smooth bed. This is confirmed by the spectra measured higher in the water column (at $z / \phi=2.5$ and 4 ), where the 


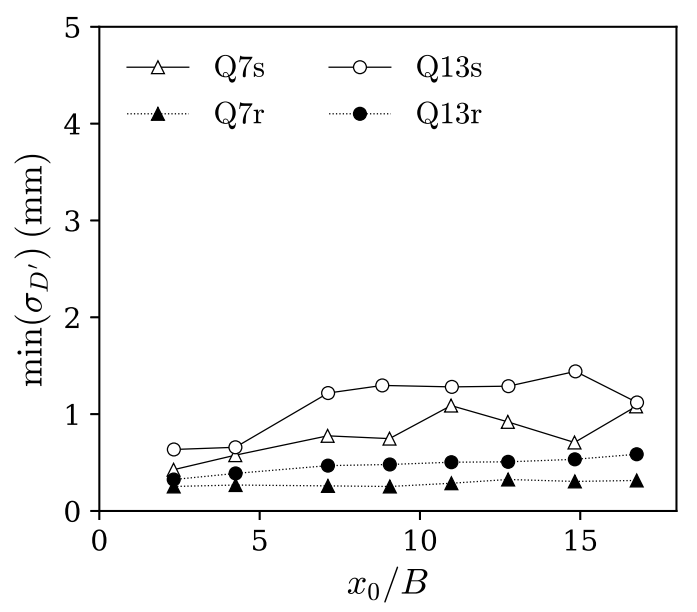

(a) $\min \left(\sigma_{D^{\prime}}\right)$

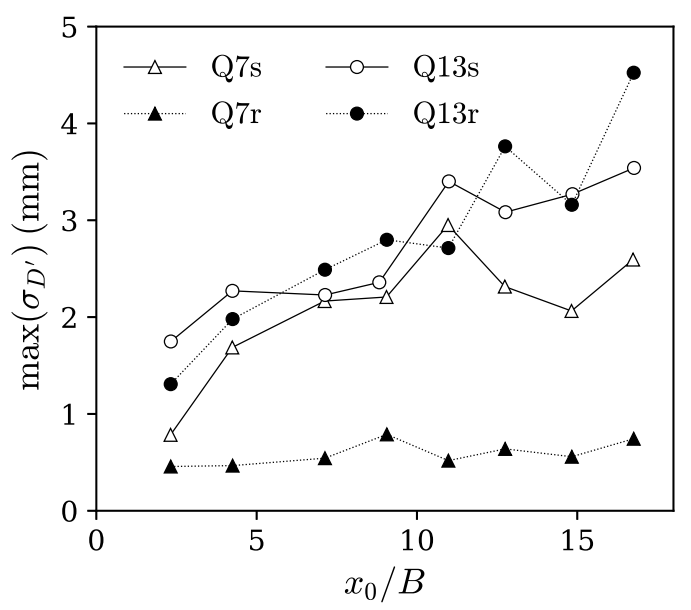

(b) $\max \left(\sigma_{D^{\prime}}\right)$

FIG. 9. Minimal (a) and maximal (b) standard deviation of the flow depth fluctuations $\sigma_{D^{\prime}}$ measured across the channel, at various longitudinal positions $x_{0} / B$. The $\max \left(\sigma_{D^{\prime}}\right)$ - and $\min \left(\sigma_{D^{\prime}}\right)$-values are observed at anti-nodes and nodes, and are associated with the predominant and secondary modes, respectively.

difference in the peak magnitude between smooth- and rough-bed cases is negligible (Figs. 10c-d) or lower than near the bed (compare Figs. 10g-h to Figs. 10e-f).

Third, the shallowness effect on the vortex shedding magnitude is very visible on the spectra of $u_{y}^{\prime}$ for the smooth-bed cases. The energy of the vortex shedding (the peak in $S_{y y}$ ) is higher for Q13s than for Q7s over the whole water column (compare Figs. 10e-g to Figs. 10a-c). This can be explained by the fact that the vortex-shedding-induced oscillating lift force acts over a shorter length of cylinder for Q7s than for Q13s (bed-induced turbulence having far less influence on the 
transverse velocity motion for smooth-bed cases than for rough-bed cases as previously shown).

As a result, the combined effects of a high rough-bed-induced turbulence (disorganizing transverse fluctuating motion and reducing the vortex shedding energy near the bed) and of an oscillating lift force acting along a small vertical distance are observed for the smaller discharge over rough bed (Q7r). This results in the absence of noticeable surface oscillations (Fig. 5b, table III) and of a predominant frequency in the spectra of $u_{y}^{\prime}$ in the fast-stream region (Figs. 10b-d, red dashed lines). Without these combined effects (for Q7s, Q7r, and Q13s), the predominant mode oscillations increase along the flume (Fig. 9b), as if there was a cumulative effect of the oscillating lift-forces (and associated transverse pressure variations) on the multiple cylinders. The comparison of the $\max \left(\sigma_{D^{\prime}}\right)$-values for Q7s, Q7r, and Q13s also shows that the effect of the bed-surface state is higher than the shallowness effect. Note that, as water depth is the same for both cases Q7s and Q7r (56 mm, see table I), this means that the energy dissipation induced by bed-friction over the rough bed is of the same order of magnitude as the energy dissipation by the surface waves.

Overall, we can thus conclude that both bed-surface state and high shallowness can alter the seiche waves, the high 3D turbulence induced by the rough bed disorganizing the transverse fluctuating flow motion in the near-bed region, and the relative weight of the oscillating lift-force associated with vortex shedding decreasing with an increasing shallowness. The combined effects of a rough bed and a high shallowness level results in the decay of the seiche waves.

\section{VELOCITY FLUCTUATIONS}

\section{A. Turbulence statistics}

The measurements in the transverse measuring plane (see Fig. 2a) of the normalized streamwise and transverse turbulence intensities, $\overline{u_{x}^{\prime 2}} / U_{Q}^{2}$ and $\overline{u_{y}^{\prime 2}} / U_{Q}^{2}$, and transverse Reynolds shear stress $\overline{u_{x}^{\prime} u_{y}^{\prime}} / U_{Q}^{2}$, are presented in Fig. 11. One cylinder is located $4 \mathrm{~cm}$ upstream of the measuring plane at $y / \phi=4$, and another cylinder is located $4 \mathrm{~cm}$ downstream of the plane at $y / \phi=12$. Transverse profiles of the measured and simulated values of $\overline{u_{x}^{\prime 2}} / U_{Q}^{2}, \overline{u_{y}^{\prime 2}} / U_{Q}^{2}$, and $\overline{u_{x}^{\prime} u_{y}^{\prime}} / U_{Q}^{2}$ at a given elevation $\left(z / \phi=3\right.$ for $71 \mathrm{~s}^{-1}$, and $z / \phi=5$ for $\left.131 \mathrm{~s}^{-1}\right)$ are also shown in Fig. 12.

For the rough-bed cases, the inhibiting effect of the bed-induced turbulence on the transverse fluctuating motion that was previously observed on the spectra in Figs. 10b and 10f, is also very clear in Fig. 11. For instance, in the upstream cylinder wake $(y / \phi \approx 4)$ below $z / \phi \approx 2$, the mea- 


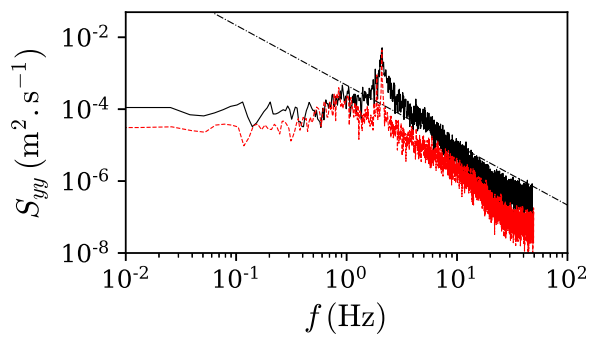

(a) Q7s, $z / \phi=1$

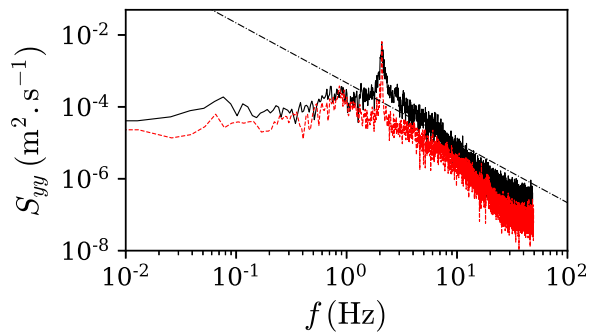

(c) $\mathrm{Q} 7 \mathrm{~s}, z / \phi=2.5$

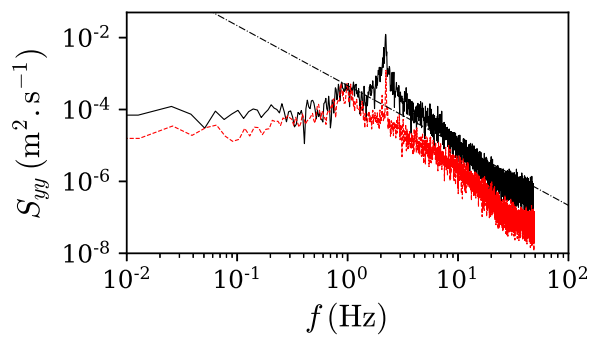

(e) Q13s, $z / \phi=1$

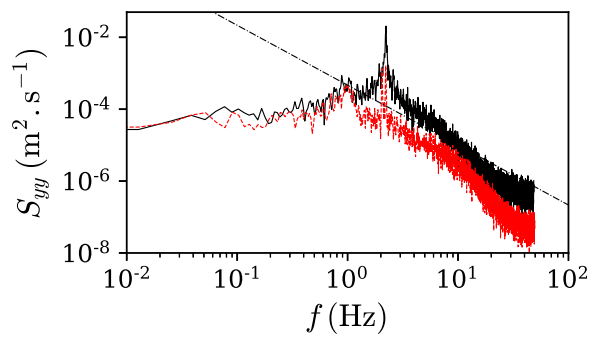

(g) Q13s, $z / \phi=4$

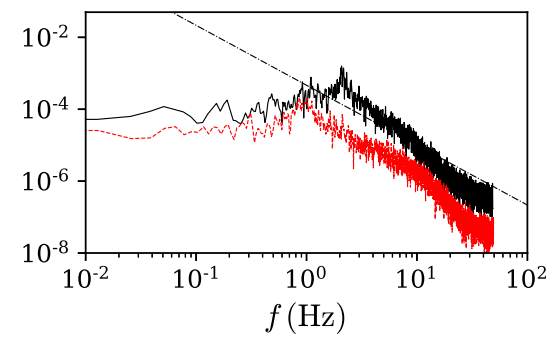

(b) Q7r, $z / \phi=1$

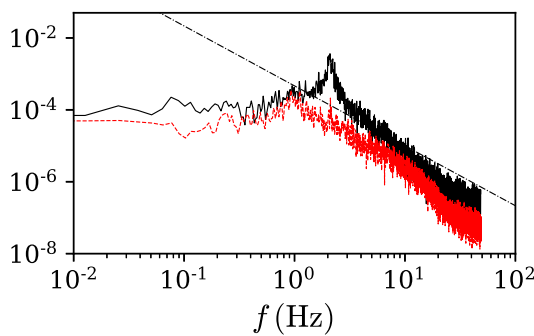

(d) $\mathrm{Q} 7 \mathrm{r}, z / \phi=2.5$

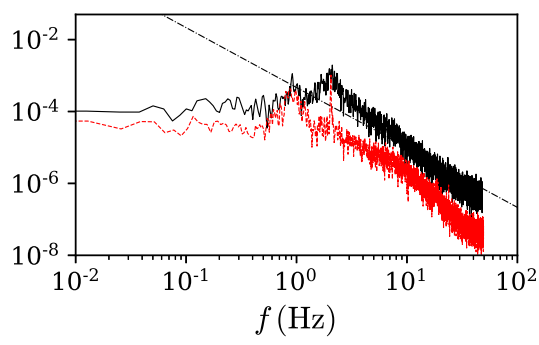

(f) Q13r, $z / \phi=1$

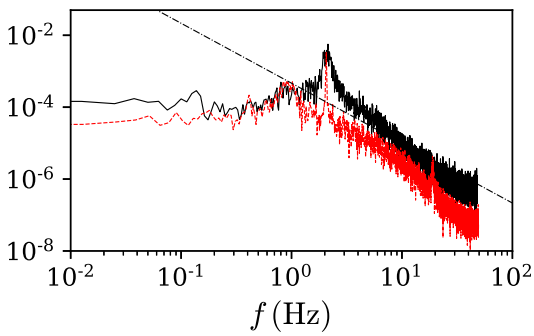

(h) Q13r, $z / \phi=4$

FIG. 10. Power density spectra $S_{y y}$ of the transverse velocity fluctuation $u_{y}^{\prime}$ downstream of a cylinder at $y / \phi=4$ (point A, see Fig. 2a) indicated by a solid black line; and spectra in the fast-stream region at $y / \phi=8$ (point B) indicated by a red dashed line. Measurements are taken near the bed at $z / \phi=1$ for cases Q7s $(a)$, Q7r $(b)$, Q13s $(e)$ and Q13r $(f)$, and higher in the water column at $z / \phi=2.5$ for cases Q7s $(c)$ and Q7r $(d)$ and at $z / \phi=4$ for cases Q13s $(g)$ and Q13r $(h)$. The mixed line indicates the classical $-5 / 3$ slope of the 3D turbulence decay in the inertial range. 
sured Reynolds shear stresses decrease downwards for Q7r and Q13r (Figs. 11b and 11d), while they are rather vertically uniform for Q7s and Q13s (Figs. 11a and 11c). In addition, over the whole water column, the region of high shear stress is wider for the smooth-bed cases than for the rough-bed cases, irrespective of the shallowness level $\left(7\right.$ or $\left.131 \mathrm{~s}^{-1}\right)$. This would suggest that bed-induced turbulence could have an impact on the vortex shedding not only in the near-bed region (bed stabilization effect as observed by Heidari et al. ${ }^{43}$ ), but actually over the whole water column. This is also observed for the turbulence intensities for $131 \mathrm{~s}^{-1}$ with a wider region of high values of $\overline{u_{x}^{\prime 2}} / U_{Q}^{2}$ and $\overline{u_{y}^{\prime 2}} / U_{Q}^{2}$ for Q13s than for Q13r (Figs. 11c,d). This would explain why the effect of the bed-surface state is stronger than the shallowness effect (as mentioned in the previous section) for the range of flow conditions studied here.

Fig. 12 highlights a very good agreement between numerical and experimental results for the two parameters involving transverse fluctuating water motion, $\overline{u_{y}^{\prime 2}}$ and $\overline{u_{x}^{\prime} u_{y}^{\prime}}$. In the upstream cylinder wake $(y / \phi \approx 4)$, the strong transverse turbulence intensities and the strong transverse gradients in Reynolds shear stress are particularly well predicted by the numerical model. The agreement between measurements and simulations is as good for case $\mathrm{Q} 7 \mathrm{r}$ with very small surface oscillations as for cases Q7s, Q13s and Q13r accompanied with noticeable surface waves. As the numerical model does not take into account the surface oscillations, these results would suggest that surface oscillations have little effect on the magnitude of velocity fluctuations (in the range of the flow configurations studied), despite the lock-in process between the frequencies of vortex shedding and surface waves.

The results of the model are less good for the streamwise turbulence intensity, $\overline{u_{x}^{\prime 2}}$, which is underestimated for both rough- and smooth-bed cases, irrespective of the $y / \phi$-position across the measuring plane. The longitudinal progressive wave going upstream might give rise to an additional fluctuating motion in the longitudinal direction, but as the $\overline{u_{x}^{\prime 2}}$-values are also underestimated for case Q7r (for which surface oscillations are very low), this explanation does not hold. On the other hand, the too small simulated $\overline{u_{x}^{\prime 2}}$-values could arise from the underestimate of the bedinduced turbulence when using a wall function in the hybrid RANS-LES approach (Eqs. 4 to 9), as observed by Chatelain and Proust ${ }^{44}$ for some flows in a two-stage (compound) open-channel. 

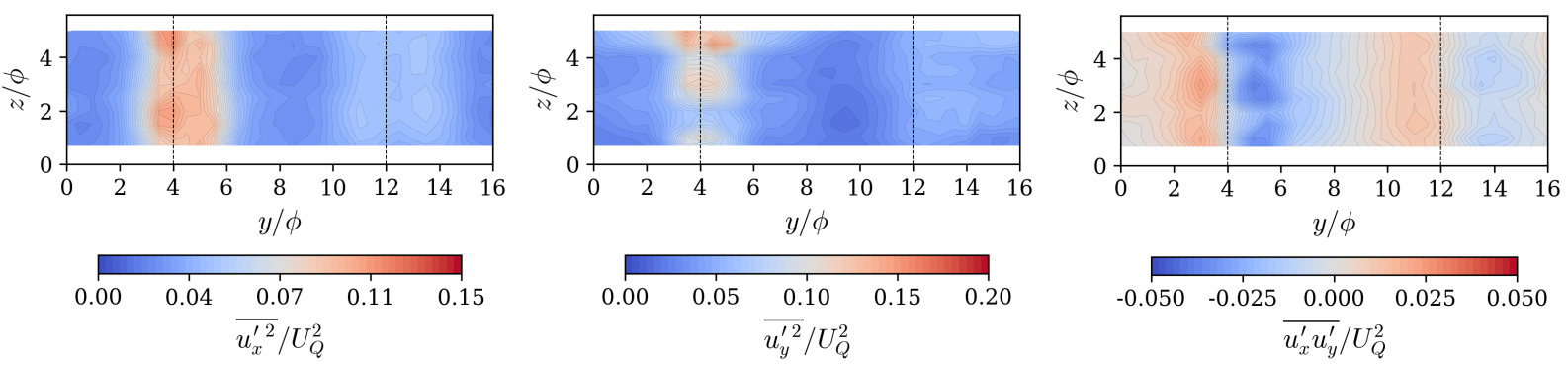

(a) Q7s
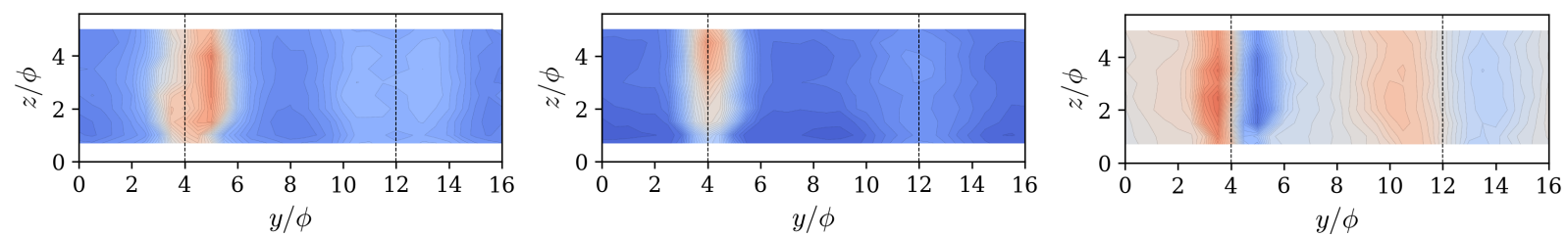

(b) Q7r
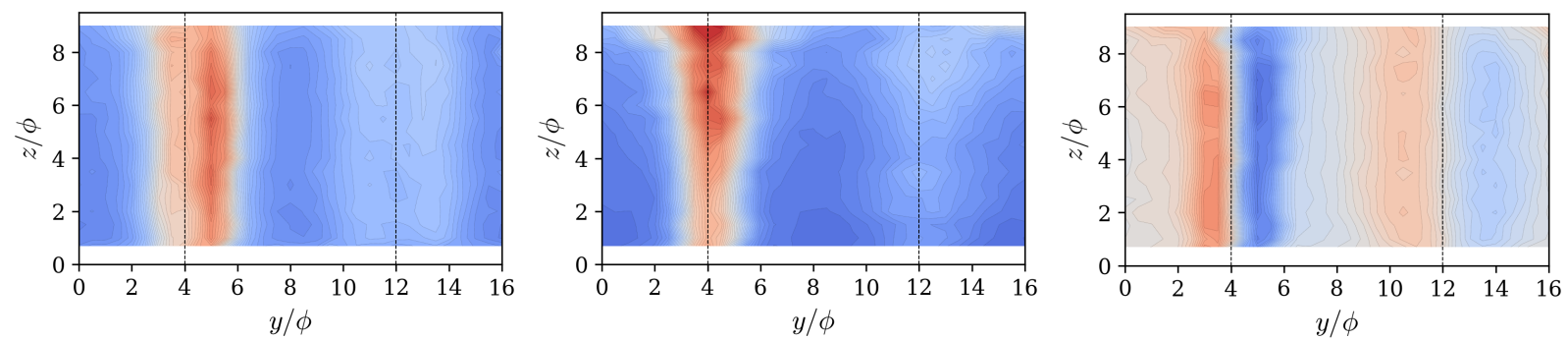

(c) Q13s
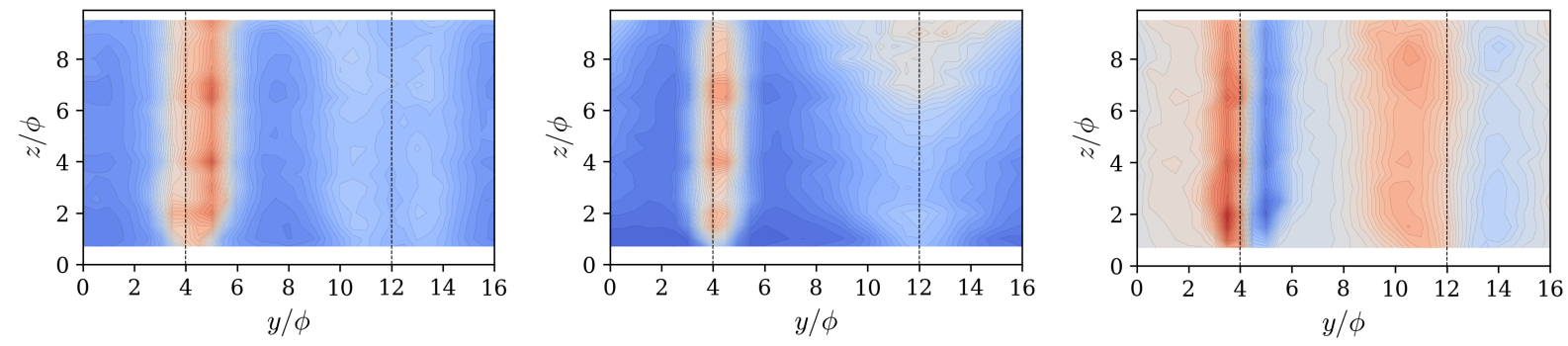

(d) Q13r

FIG. 11. Cross-sectional distribution of the measured streamwise and spanwise turbulence intensities, $\overline{u_{x}^{\prime 2}} / U_{Q}^{2}$ and $\overline{u_{y}^{\prime 2}} / U_{Q}^{2}$, and transverse Reynolds shear stress $\overline{u_{x}^{\prime} u_{y}^{\prime}} / U_{Q}^{2}$ for cases Q7s $(a)$, Q7r $(b)$, Q13s $(c)$ and Q13r $(d)$. The dashed lines indicate the spanwise positions of the upstream cylinder at $y / \phi=4$ and downstream cylinder at $y / \phi=12$ (see Fig. 2a) 

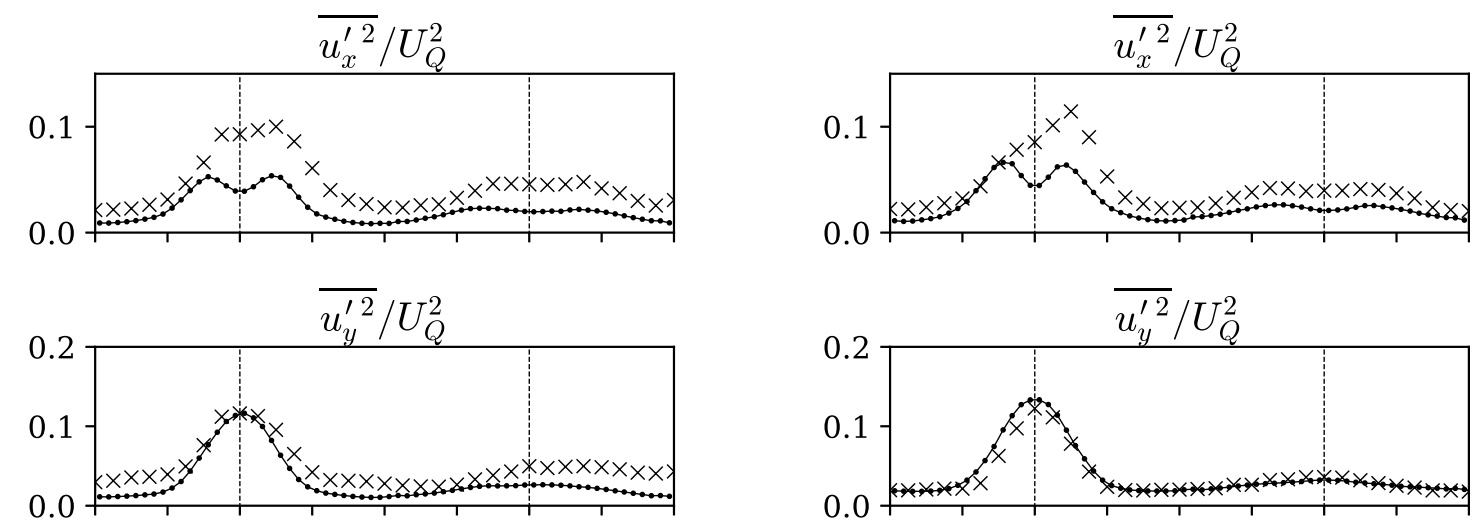

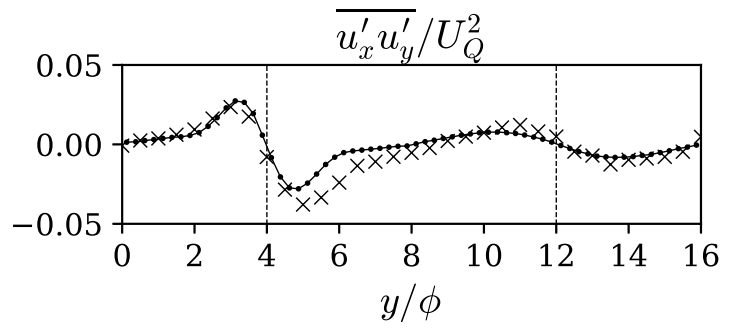

(a) Q7s
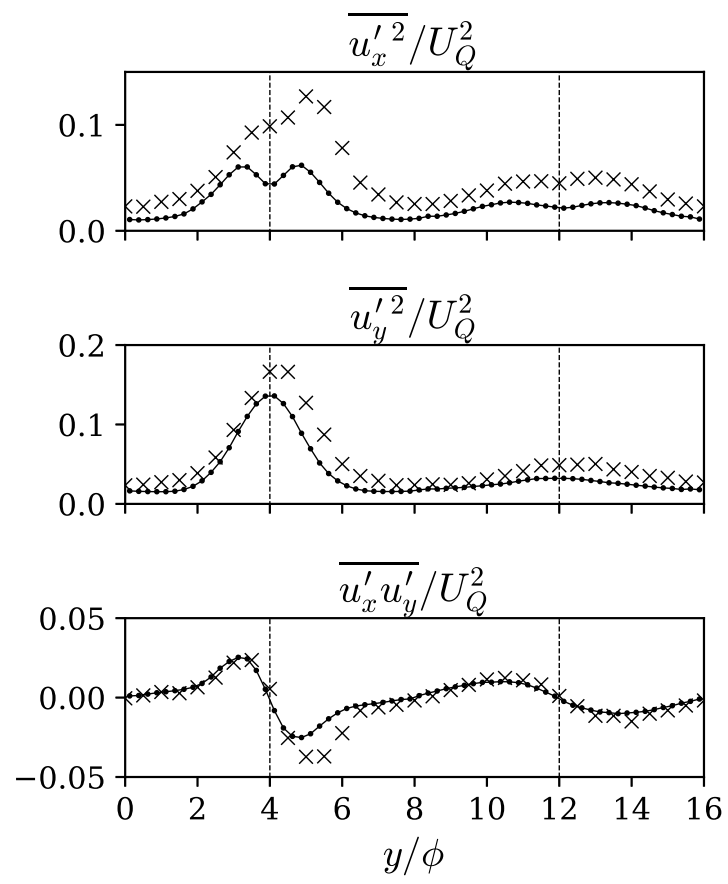

(c) Q13s

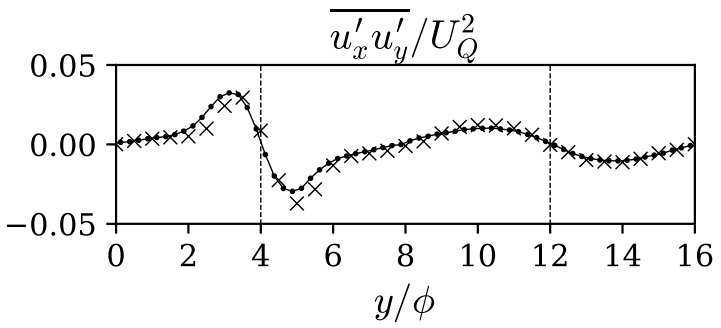

(b) $\mathrm{Q} 7 \mathrm{r}$

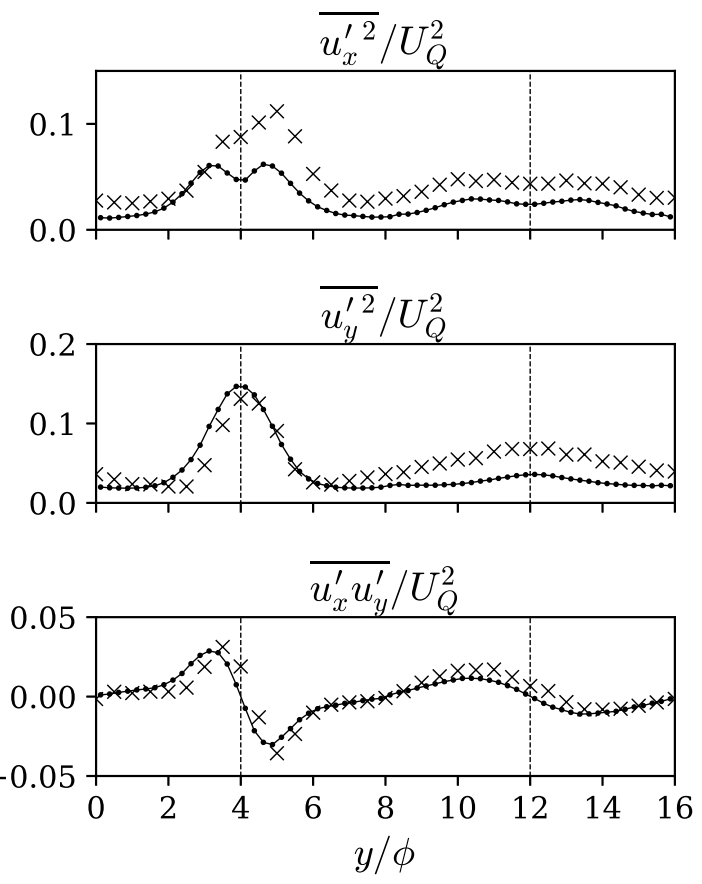

(d) Q13r

FIG. 12. Transverse profiles of the streamwise and spanwise turbulence intensities, $\overline{u_{x}^{\prime 2}} / U_{Q}^{2}$ and $\overline{u_{y}^{\prime 2}} / U_{Q}^{2}$, and of the transverse Reynolds shear stress $\overline{u_{x}^{\prime} u_{y}^{\prime}} / U_{Q}^{2}$ : experimental data $(\times)$ and numerical results $(\bullet)$ at $z / \phi=3$ for cases Q7s $(a)$ and Q7r $(b)$, and at $z / \phi=5$ for cases Q13s $(c)$ and Q13r $(d)$. 


\section{B. Predominant frequency and Strouhal number}

From the power density spectra of velocity fluctuation $u_{y}^{\prime}$ (see Fig. 10), the predominant frequency of the transverse fluctuating flow motion can be determined at each position $y / \phi$. Transverse profiles of the measured frequency (denoted as $f_{u_{y}^{\prime}}$ ) are presented in Fig. 13. The measured and simulated $\left(f_{u_{y}^{\prime}}^{n u m}\right)$ frequencies downstream of a cylinder (at point A in Fig. 2a) are also given in table IV, along with the associated Strouhal numbers $S_{\mathrm{t}}=\left(f_{u_{y}^{\prime}} \phi\right) / U_{Q}$ and $S_{\mathrm{t}}^{\text {num }}=\left(f_{u_{y}^{\prime}}^{\text {num }}\right) \phi / U_{Q}^{\text {num }}$.

In the upstream cylinder wake (table IV), the measured vortex shedding frequency $\left(f_{u_{y}^{\prime}}\right)$ and the measured Strouhal number $\left(S_{t}\right)$ are lower than the simulated ones. In the experiments, owing to the lock-in process between vortex shedding and transverse surface waves, the values of $f_{u_{y}^{\prime}}$ are very close to the surface waves frequencies $f_{D^{\prime}}$ associated with the predominant mode observed in the downstream part of the flume, i.e., $n=7$ for cases Q7s, Q7r and Q13s and $n=6$ for Q13r (see table III). On the other hand, as the numerical model neglects the surface oscillation effect (rigid lid assumption), the $S_{t}^{\text {num }}$-values range from 0.191 to 0.197 , in agreement with the dimensionless vortex shedding frequency observed behind a single circular cylinder set in an unbounded laterally flow, for the range of the Reynolds numbers studied here ${ }^{45}$. Interestingly, the experimental Strouhal numbers are smaller than the computed ones, and close to 0.16 . In a comprehensive experimental study on surface waves, Viero, Pradella, and Defina ${ }^{42}$ found out that two distinct resonance conditions can coexist for the same wave mode: the first one corresponds to that of an isolated cylinder $\left(S_{t}=0.2\right.$ in their experiments and in the present simulations $)$ and the second one is observed at a smaller Strouhal number. Their experimental results showed that, for mode $n=1$, the secondary resonance occurred at $S_{t}=0.16$ for a staggered array of cylinders and at $S_{t}=0.145$ for an in-line distribution of cylinders. The present experimental results could thus reflect a secondary resonance for modes 6 or 7 , at $S_{t} \approx 0.16$. Relying on the results of Viero, Pradella, and Defina $^{42}$, higher flow rates should have been tested to confirm the existence of a primary resonance (leading to a mode 9 in our study). Unfortunately, this was not possible because of the limited height of the sidewalls $(11.5 \mathrm{~cm}$, see Fig. 4 the videos the smooth-bed cases).

Across the channel, for the higher discharge $\left(131 \mathrm{~s}^{-1}\right)$, the distributions of $f_{u_{y}^{\prime}}$ (Fig. 13) and $f_{D^{\prime}}\left(\right.$ Fig. 5, data at $\left.x_{0} / B=12.8\right)$ are strongly linked. For case Q13s, the frequencies $f_{D^{\prime}}$ that are associated with the predominant and secondary modes of surface oscillation (Fig. 5c) are retrieved along the profile of $f_{u_{y}^{\prime}}$ (Fig. 13c), but with opposite distributions. At surface nodes, the higher $f_{u_{y}^{\prime}}$-value and the lower $f_{D^{\prime}}$-value are observed $\left(y / \phi \approx 4\right.$ and $\left.y_{0} / B \approx 0.5\right)$, while the opposite result 


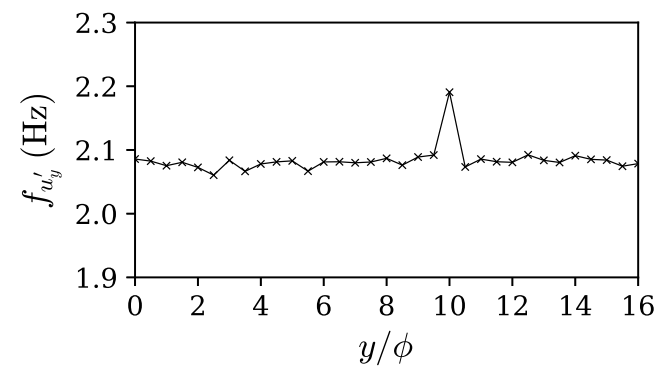

(a) Q7s

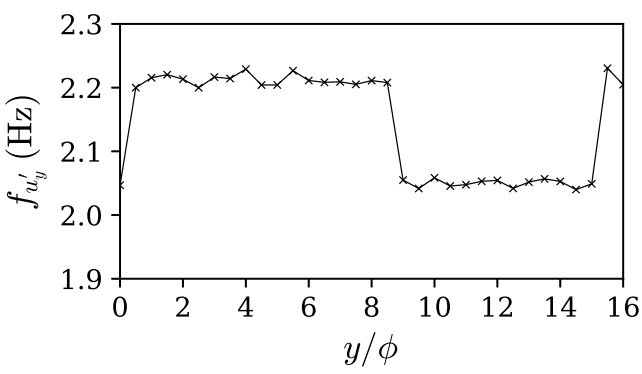

(c) Q13s

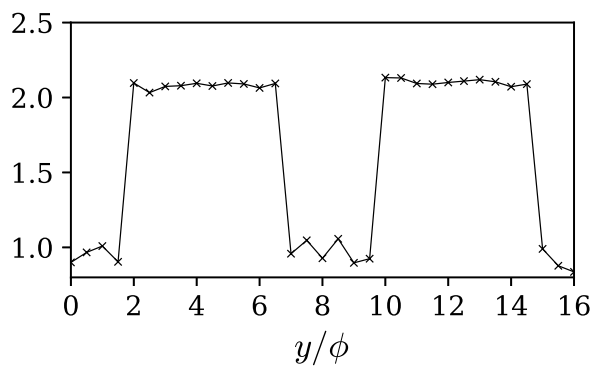

(b) Q7r

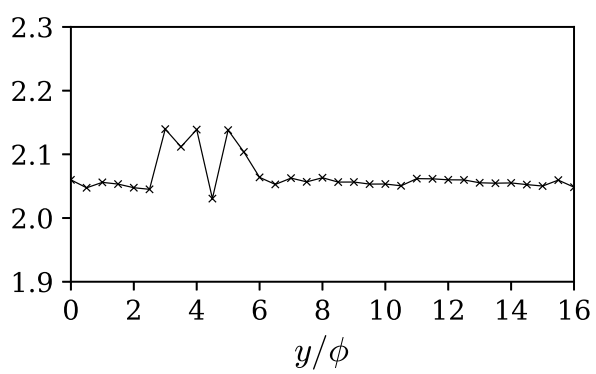

(d) Q13r

FIG. 13. Transverse profiles of the main frequency of the transverse fluctuating motion, $f_{u_{y}^{\prime}}$, determined from the power density spectra of transverse velocity fluctuation $u_{y}^{\prime}$ (see Fig. 10). Measurements are taken at longitudinal position $x / \phi=4$, and at elevation $z / \phi=2.5$ for cases Q7s $(a)$ and Q7r $(b)$ and $z / \phi=4$ for cases Q13s (c) and Q13r (d).

can be observed at anti-nodes $\left(y / \phi \approx 12\right.$ and $\left.y_{0} / B \approx 0.58\right)$. For case Q13r, $f_{u_{y}^{\prime}}$ is almost constant in the transverse profile (Fig. 13d), around a value of $2.07 \mathrm{~Hz}$, as observed for $f_{D^{\prime}}$ (Fig. 5d).

For the smaller discharge, the link between the distributions of $f_{D^{\prime}}$ and $f_{u_{y}^{\prime}}$ is not as strong. For case Q7s, the predominant mode is visible in both distributions, but the secondary mode is visible in the $f_{D^{\prime}}$-distribution only. For case Q7r, a constant frequency has been observed in the cylinders wakes regions $(2 \leq y / \phi \leq 6.5$ and $10 \leq y / \phi \leq 14.5)$, around $2.09 \mathrm{~Hz}$. Outside these regions, in the free-stream, a lower value $(1 \mathrm{~Hz})$ has been observed, but this frequency is not associated with a noticeable peak on the spectra in Figs. 10b,d.

\section{TIME-AVERAGED FLOW STRUCTURE}

The structure of the time-averaged flow in the longitudinal and transverse measuring planes (see Fig. 2) is depicted in Figs. 14 to 18. The measured and simulated time-averaged streamwise velocity $\overline{u_{x}} / U_{Q}$ in the longitudinal plane are shown in Fig. 14. The measured velocities $\overline{u_{x}} / U_{Q}$ 
TABLE IV. Predominant frequency of the transverse velocity fluctuation, $u_{y}^{\prime}$, behind a cylinder (point $\mathrm{A}$ in Fig. 2a): $f_{u_{y}^{\prime}}$ and $f_{u_{y}^{\prime}}^{n u m}$ are the measured and simulated frequencies, respectively; and $\mathrm{S}_{\mathrm{t}}=\left(f_{u_{y}^{\prime}} \phi\right) / U_{Q}$ and $\mathrm{S}_{\mathrm{t}}^{\text {num }}=f_{u_{y}^{\prime}}^{\text {num }} \phi / U_{Q}^{\text {num }}$ are the two associated Strouhal numbers.

\begin{tabular}{llllc}
\hline \hline Case & $f_{u_{y}^{\prime}}$ & $f_{u_{y}^{\prime}}^{\text {num }}$ & $\mathrm{S}_{\mathrm{t}}$ & $\mathrm{S}_{\mathrm{t}}^{\text {num }}$ \\
& $(\mathrm{Hz})$ & $(\mathrm{Hz})$ & $(-)$ & $(-)$ \\
\hline Q7s & 2.08 & 2.43 & 0.166 & 0.194 \\
Q7r & 2.09 & 2.46 & 0.167 & 0.197 \\
Q13s & 2.23 & 2.62 & 0.163 & 0.191 \\
Q13r & 2.14 & 2.58 & 0.163 & 0.197 \\
\hline \hline
\end{tabular}

in the transverse plane are shown in Fig. 15. The vertical profiles of the measured and simulated velocities $\overline{u_{x}} / U_{Q}$ at points A, B and C (Fig. 2) are reported in Fig. 16. The measured and simulated transverse and vertical velocity fields in the transverse measuring plane are shown in Fig. 17. Lastly, transverse profiles of the measured and simulated transverse and vertical velocities, close to the bottom at $z / \phi=1$, are presented in Fig. 18 .

\section{A. Secondary currents and streamwise velocity-bulge in the near-bed region}

In the cylinder wake, outside the reverse flow region (defined by negative $\overline{u_{x}}$-values), an acceleration of the flow can be observed near the bed for all cases in the experiments and simulations (Fig. 14). This acceleration results in a local peak of $\overline{u_{x}}$, also called near-bed velocity-bulge (section I). The peak of $\overline{u_{x}}$ is visible along the vertical profiles of $\overline{u_{x}}$ at point A (Fig. 16), and also in Fig. 14 for the simulated results, where velocity $\overline{u_{x}} / U_{Q}$ is computed in the longitudinal plane over the entire flow depth until the bottom.

This flow acceleration is caused by the helical secondary currents with a longitudinal axis (socalled 'base vortices', see section I) that develop near the bed. The two counter-rotating cells can be seen in Fig. 17 from either side of $y / \phi=12$, i.e. $12 \mathrm{~cm}$ downstream of a cylinder. The early stage of development of these vortices is visible at $y / \phi=4(4 \mathrm{~cm}$ downstream of a cylinder $)$ in Fig. 17 and Fig. 18, where a noticeable upward motion can be observed behind the cylinder, along with strong spanwise velocities directed towards the cylinder from either side of $y / \phi=4$.

The base vortices bring high-momentum fluid from the high-speed region into the cylinder 
wake (Fig. 17), resulting in the near-bed velocity-bulge (Fig. 14). The base vortices have also an impact in the high-speed region, as shown in Fig. 15 (at $y / \phi=8$ ) and Fig. 16 (profiles at point B). A noticeable flow deceleration when approaching the water surface can be observed for the two shallower cases Q7s and Q7r. Though less noticeable, this deceleration is also visible for cases $\mathrm{Q} 13$ s and Q13r near $z / \phi \approx 4$. The base vortices are responsible for this flow deceleration, as they bring low-momentum fluid from the near-bed wake region to the high-speed region at $y / \phi=8$ (Fig. 17). Note that the flow deceleration in this region is predicted by the numerical model for all cases (Fig. 16, profiles at point B).

Based on the measurements in the longitudinal plane (Fig. 14), for each flow rate, the near-bed flow acceleration (or velocity-bulge) is stronger for the rough-bed case than for the smooth-bed case. The measured velocity field in the transverse plane (Fig. 17) also suggests that the base vortices are better developed for the rough-bed cases (in accordance with the stronger velocitybulge). However, as the present results are in agreement with Liu et al. ${ }^{3}$, but not with Dupuis et $a l .{ }^{5}$, we decided to further analyze the effect of the bed-surface state on the flow structure relying on simulations with a wide range of bed roughness heights (see section VI.B).

Finally, it must be recalled that Huai et al. ${ }^{46}$, who performed LESs of flows through a staggered array of rigid emergent cylinders over smooth bed, observed a second peak of mean streamwise velocity near the surface, associated with near-surface cells (in addition to the base vortices). Near-surface cells can also be observed in our simulations (see Fig. 17 at $y / \phi=4$ ), but they are small compared to the base vortices and they quickly vanish in the longitudinal direction as they are not visible at $y / \phi=12$ (12 cm downstream of the cylinder). The existence of near-surface cells cannot not be confirmed experimentally, given the limitations of the ADV probe (absence of vertical velocity measurements in the near-surface region). However, it might be assumed that the development of such structures might be disturbed by the strong free-surface oscillations observed for cases Q7s, Q13s and Q13r.

\section{B. Effect of the roughness height on the velocity bulge and upward flow}

Numerical simulations have been performed for each flow rate for (i) a smooth bed and (ii) a rough bed with a variable $k_{s}$-value ranging from 0.6 to $10 \mathrm{~mm}$. Fig. 19 presents the longitudinal evolution of the magnitude of the velocity-bulge and upwash flow. The velocity-bulge magnitude is defined at each $x / \phi$-position as the difference between the local peak velocity (denoted 


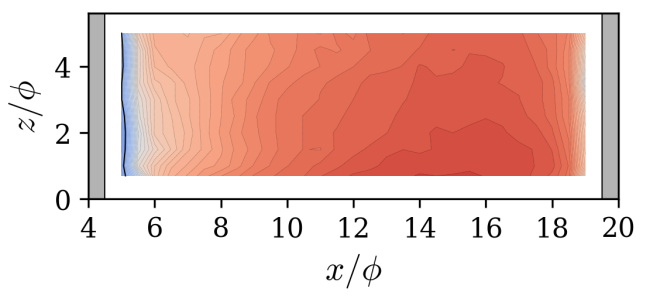

(a) Q7s, experiment

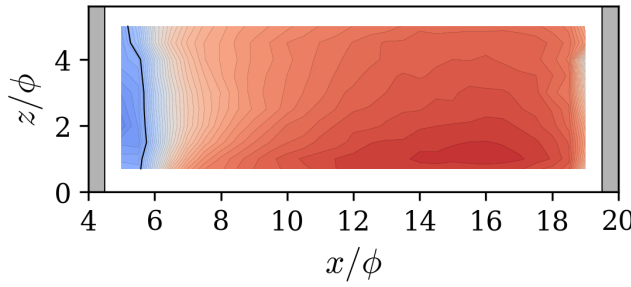

(c) Q7r, experiment

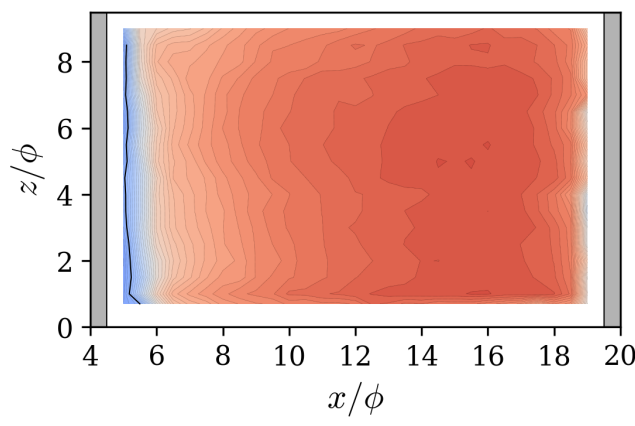

(e) Q13s, experiment

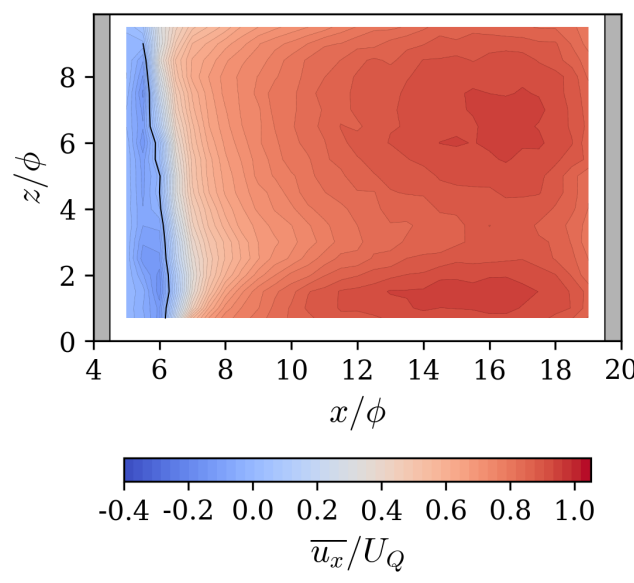

(g) Q13r, experiment

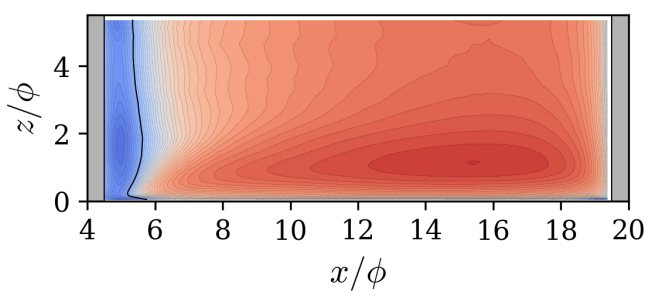

(b) Q7s, simulation

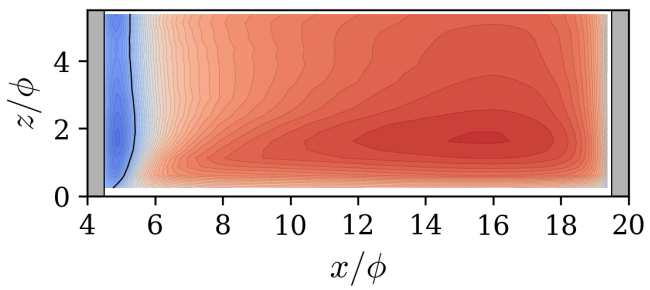

(d) Q7r, simulation

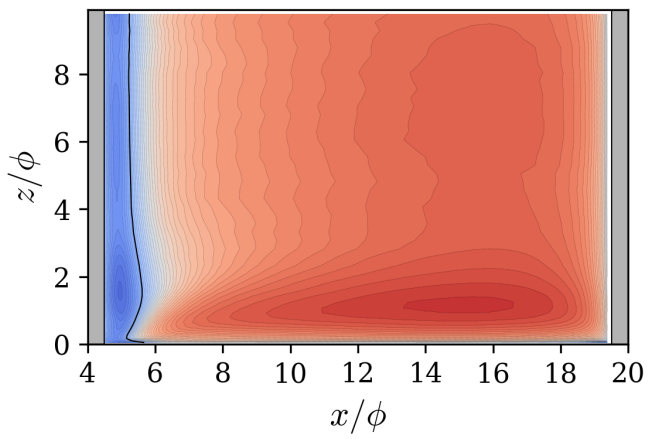

(f) Q13s, simulation
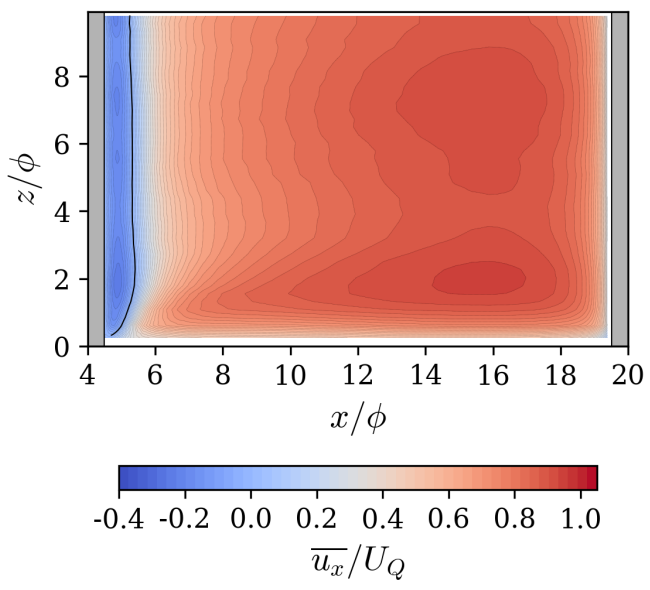

(h) Q13r, simulation

FIG. 14. Measured (left) and simulated (right) time-averaged streamwise velocity $\overline{u_{x}} / U_{Q}$ in the longitudinal measuring plane (Fig. 2) for cases Q7s $(a-b)$, Q7r $(c-d)$, Q13s $(e-f)$ and Q13r $(g-h)$. The upstream and downstream cylinders are materialized by grey rectangles. The reverse flow region, which is defined by negative $\overline{u_{x}}$-values, is bounded by a solid line. 


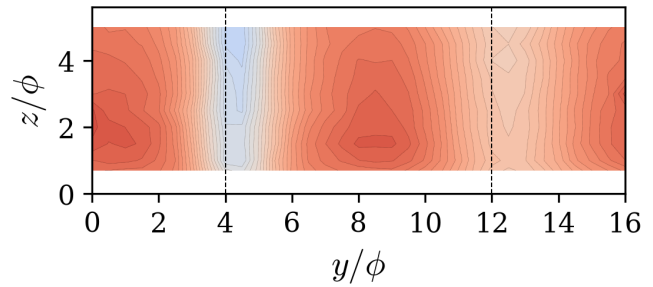

(a) Q7s

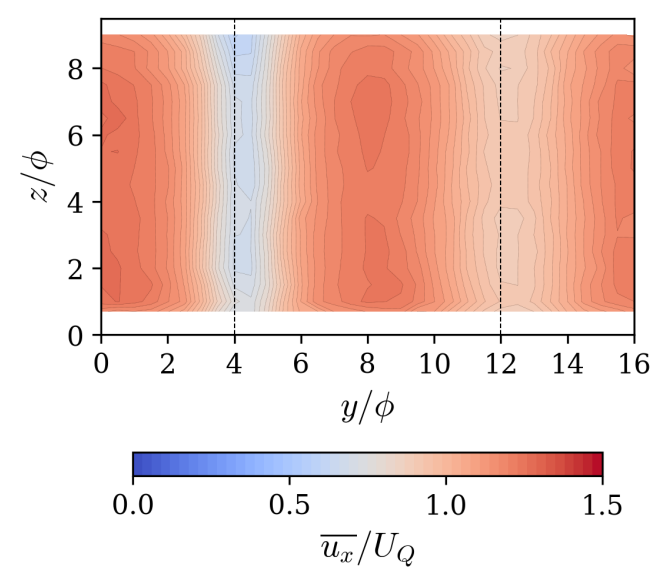

(c) Q13s

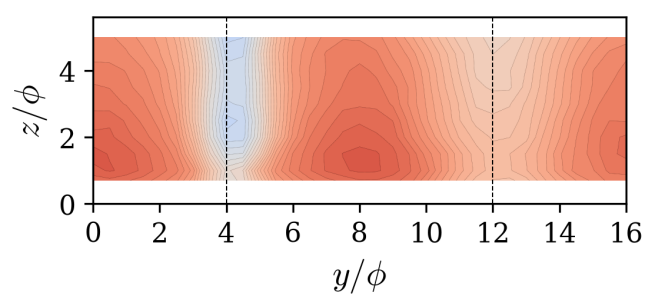

(b) Q7r

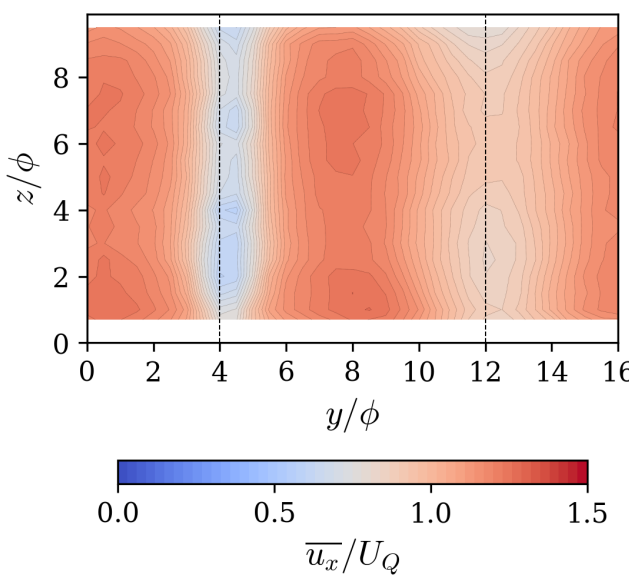

(d) Q13r

FIG. 15. Measured time-averaged streamwise velocity $\overline{u_{x}} / U_{Q}$ in the transverse measuring plane (Fig. 2) for cases Q7s (a), Q7r (b), Q13s (c) and Q13r $(d)$. The dashed lines indicate the spanwise positions of the upstream cylinder (at $y / \phi=4)$ and downstream cylinder (at $y / \phi=12$ ).

as ${\overline{u_{x}}}^{\text {bulge }}$ ) and the mean velocity in the (quasi-) Constant Velocity Region $(\mathrm{CVR})^{5}$ (denoted as ${\overline{u_{x}}}^{C V R}$ ), normalized by the bulk velocity $U_{Q}$. The upwash flow magnitude (denoted as $\max \left(\overline{u_{z}}\right)$ ) is defined at each $x / \phi$-position as the maximum mean vertical velocity along a vertical profile of $\overline{u_{z}}$, scaled by the bulk velocity. At first glance, we can notice that the magnitudes of velocity-bulge or upwash flows are comparable for the five bed-surface states studied. The same result is obtained for their longitudinal evolution between two cylinders.

For the flows over rough bed, the velocity-bulge magnitude actually increases with the $k_{s}$-value, and can reach 30 to $35 \%$ of $U_{Q}$ for the highest roughness height. In a similar way, the upwash flow also increases with the $k_{s}$-value. An increase of the bed roughness would result in a stronger near-bed velocity gradient and in more bent von Karman vortices ${ }^{15}$, which in turn would give rise to stronger base vortices. The bed roughness effect is similar to the effect of the approaching boundary layer thickness observed for single cylinders ${ }^{15-18}$. In addition, in the cylinder-nearwake, the decay of the velocity-bulge and upwash flow is faster with a higher roughness height. 

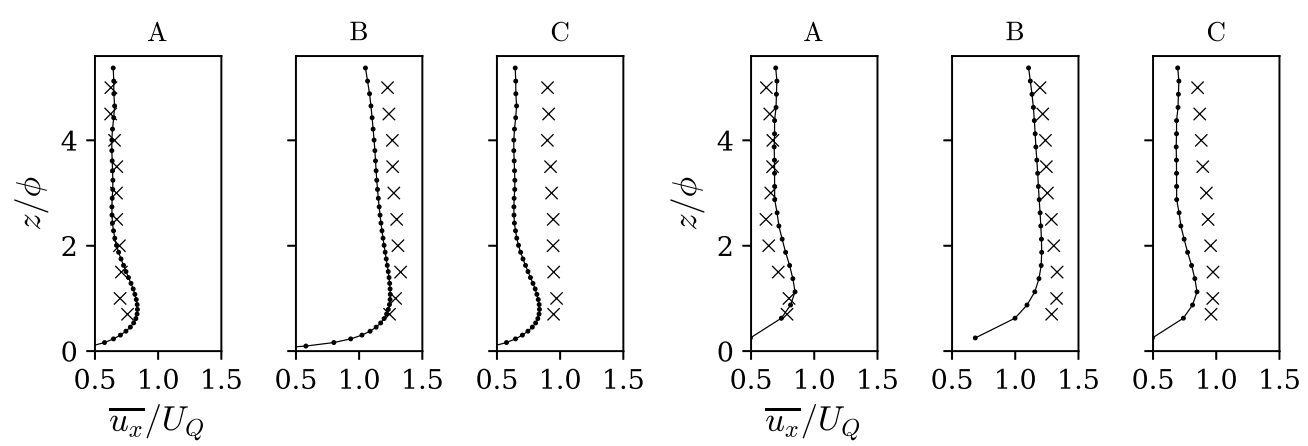

(a) Q7s

(b) Q7r
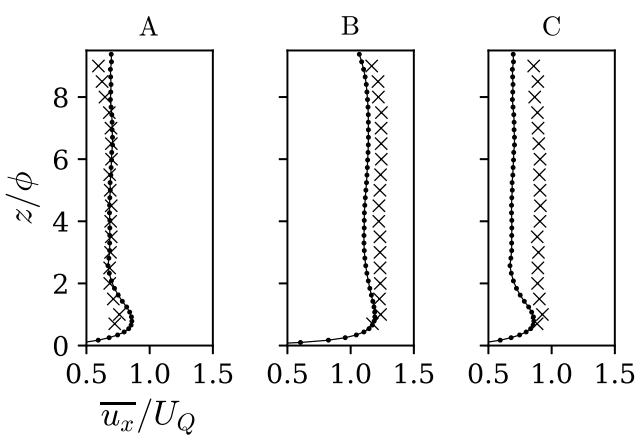

(c) Q13s
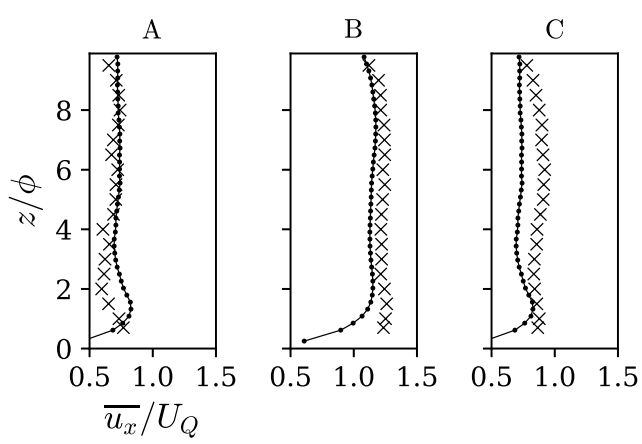

(d) Q13r

FIG. 16. Vertical profiles of the time-averaged streamwise velocity $\overline{u_{x}} / U_{Q}$ across the channel at measuring points $\mathrm{A}(4 \mathrm{~cm}$ downstream of a cylinder, at spanwise position $y / \phi=4), \mathrm{B}$ (free stream region, at $y / \phi=8$ ) and $\mathrm{C}(4 \mathrm{~cm}$ upstream of a cylinder, at $y / \phi=12)$ : experimental data $(\times)$ and numerical results $(\bullet)$ for cases Q7s $(a), \mathrm{Q} 7 \mathrm{r}(b), \mathrm{Q} 13 \mathrm{~s}(c)$ and Q13r $(d)$.

As observed in section $\mathrm{V}$, the rough-bed-induced turbulence disorganizes the transverse fluctuating flow motion and reduces the vortex shedding magnitude near the bed. We can thus assume that a rougher bed would produce a higher bed-induced turbulence, which in turn would have a higher damping effect on the von Karman vortices near the bed, resulting in a faster decay along $x$-axis.

For the flows over smooth bed, in the cylinder-near-wake, the numerical simulations also predict noticeable magnitudes of velocity-bulge and upwash flows, which are comparable to the magnitudes obtained for $k_{s}=6$ and $7 \mathrm{~mm}$, values close to the roughness height of the dense artificial grass. This result is not consistent with the measured velocity field in the transverse plane (Fig. 17) and along a transverse profile of vertical velocity $\overline{u_{z}}$ (Fig. 18), when comparing case Q7s to Q7r or Q13s to Q13r. First, this could be explained by the too slow decay of the base vortices along $x$-axis in the simulations, as shown e.g. by the vertical velocity profiles at point $\mathrm{C}$ in Fig. 16, i.e. $12 \mathrm{~cm}$ 

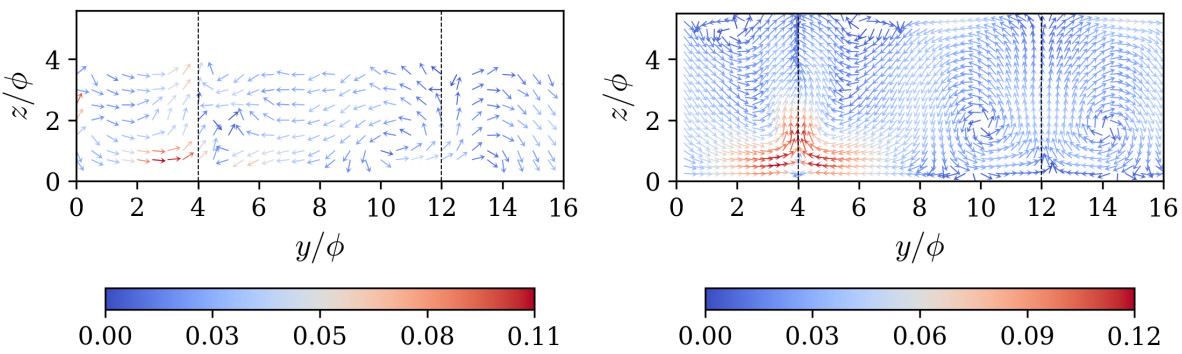

(a) Q7s, experiment

(b) Q7s, simulation
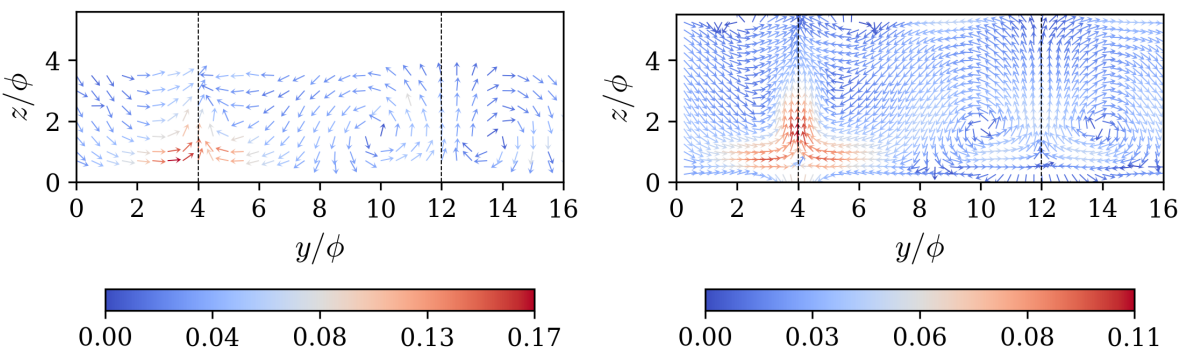

(c) Q7r, experiment

(d) Q7r, simulation
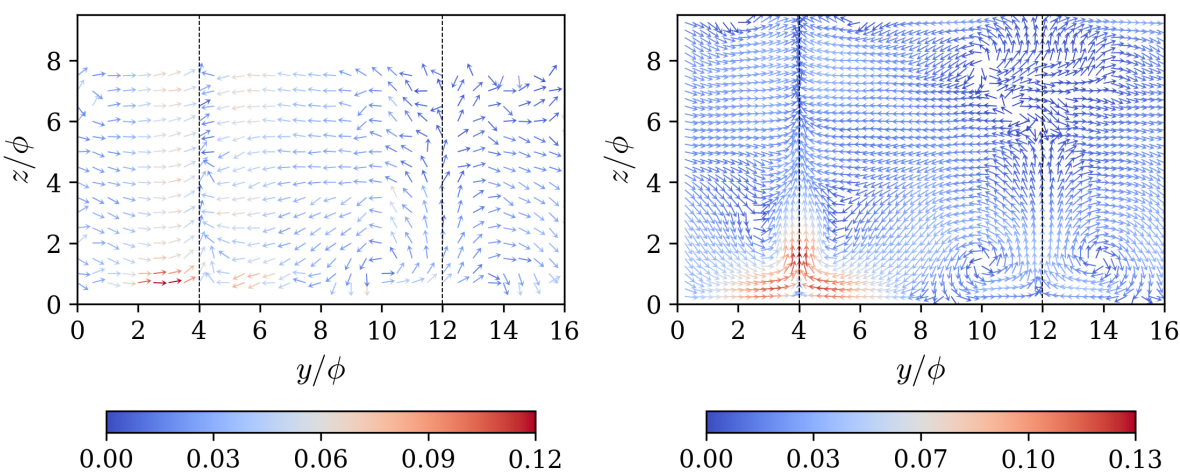

(e) Q13s, experiment

(f) Q13s, simulation

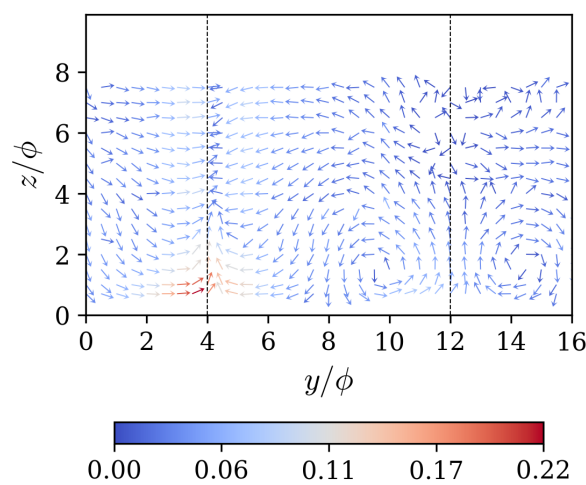

(g) Q13r, experiment

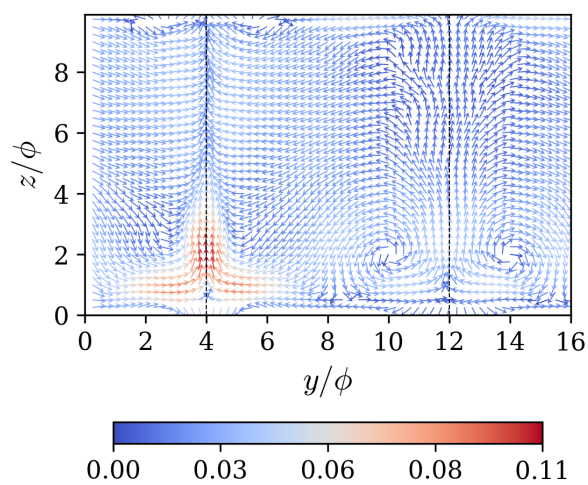

(h) Q13r, simulation

FIG. 17. Experimental (left) and simulated (right) vector fields in the transverse measuring plane for cases Q7s $(a-b)$, Q7r $(c-d)$, Q13s $(e-f)$ and Q13r $(g-h)$. The colorbars indicate the dimensionless magnitude of the in-plane velocity components $\sqrt{{\overline{u_{y}}}^{2}+{\overline{u_{z}}}^{2}} / U_{Q}$, and the dashed lines indicate the spanwise positions of the cylinders. 

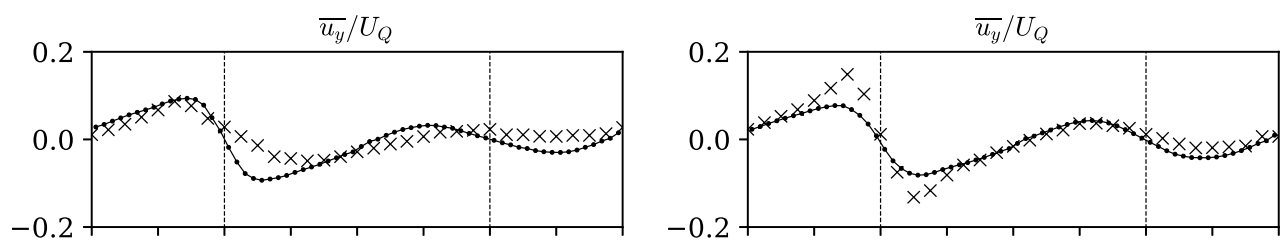

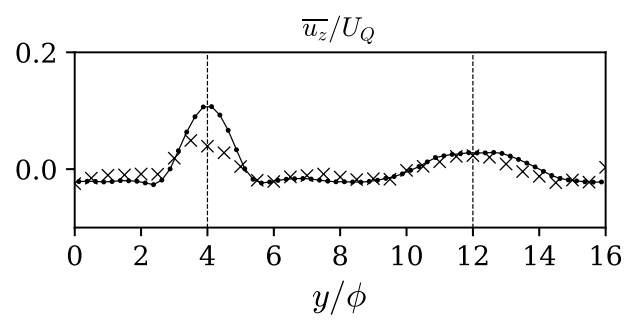

(a) Q7s
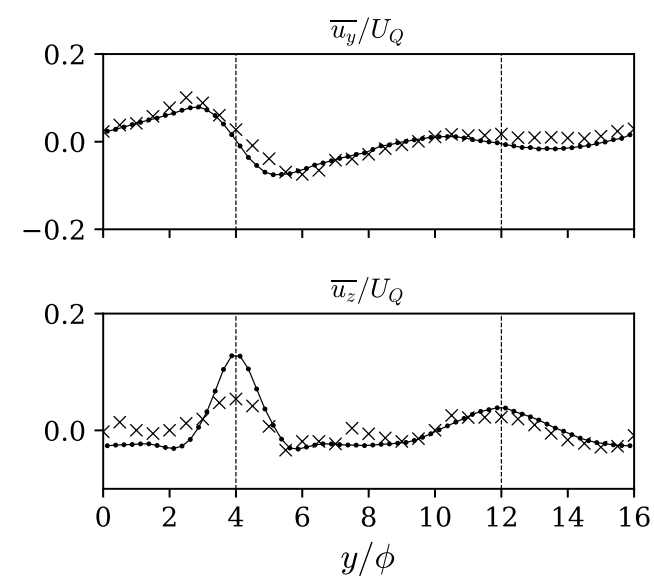

(c) Q13s

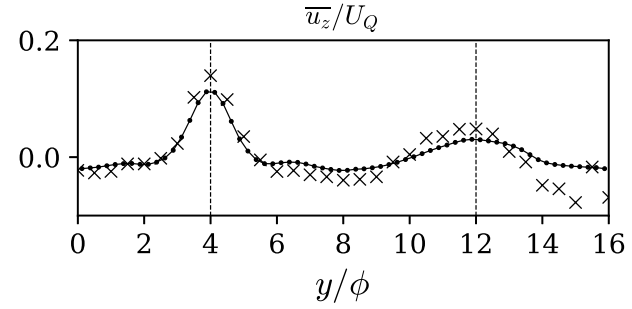

(b) Q7r
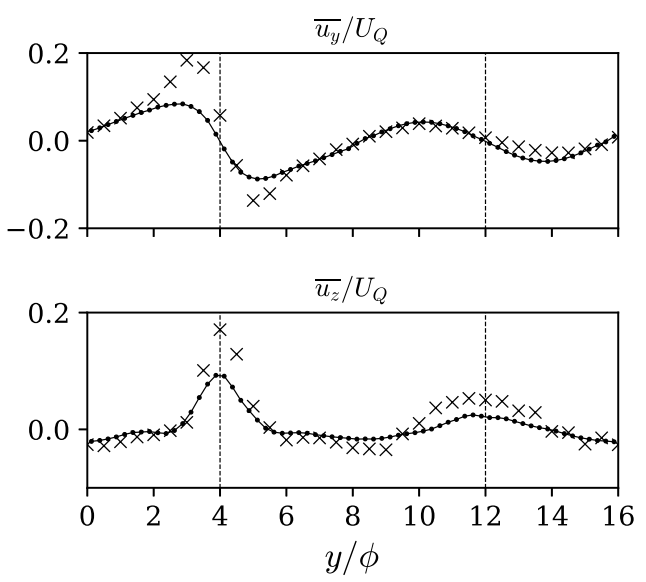

(d) Q13r

FIG. 18. Transverse profiles of the dimensionless time-averaged transverse and vertical velocities, $\overline{u_{y}} / U_{Q}$ and $\overline{u_{z}} / U_{Q}$, at elevation $z / \phi=1$ : experimental data $(\times)$ and numerical results $(\bullet)$ for cases Q7s $(a)$, Q7r (b), Q13s $(c)$ and Q13r $(d)$.

downstream of a cylinder. This too slow decay can induce a 'snowball' effect: the base vortices are responsible for the near-bed velocity-bulge, which in-turn can induce a tilting of the von-Karman vortices and larger base vortices behind the next cylinder (Figs. 16 and 19). Second, the slower decay of the base vortices in the simulations could also be due to the underestimate of the bedinduced turbulence across the channel, as observed for all cases in Fig. 12 (data of $\overline{u_{x}^{\prime 2}} / U_{Q}^{2}$ ). Last, the magnitude of the velocity-bulge and upwash flow rapidly decreases in the near-cylinder-wake, and depending on the $x$-position, this magnitude is stronger for a rough-bed than a smooth bed, or inversely. All these reasons might explain the too strong simulated base vortices, and also why 

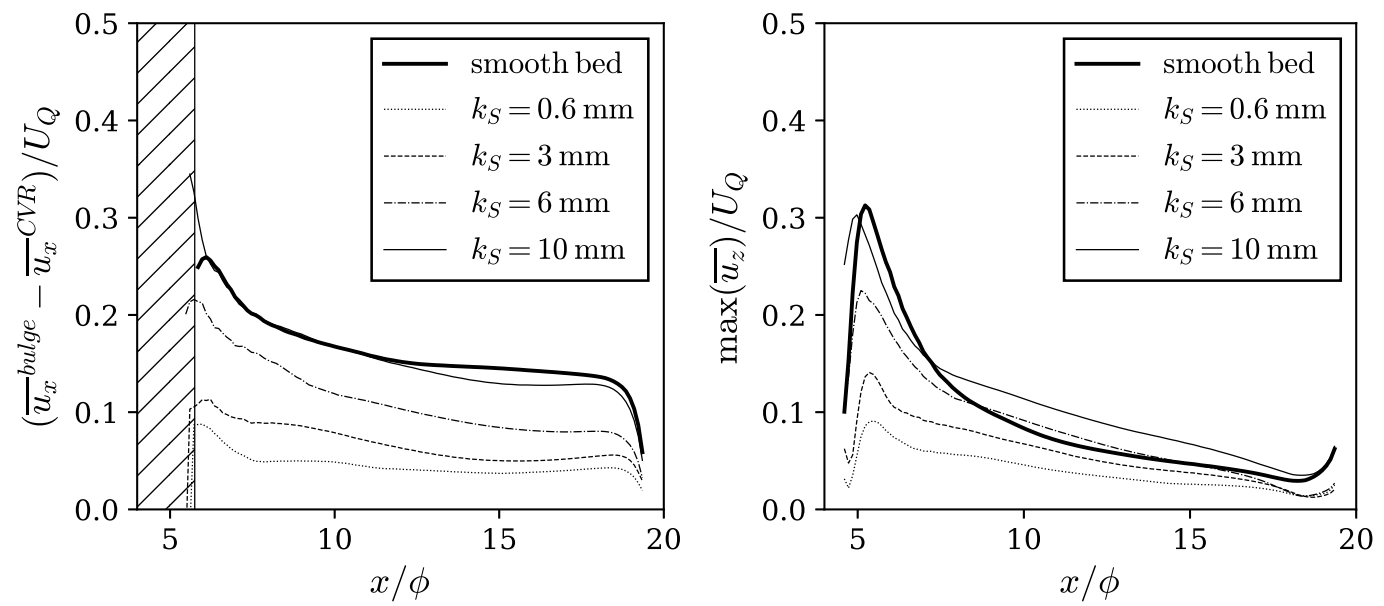

(a) $Q=71 \mathrm{~s}^{-1}$
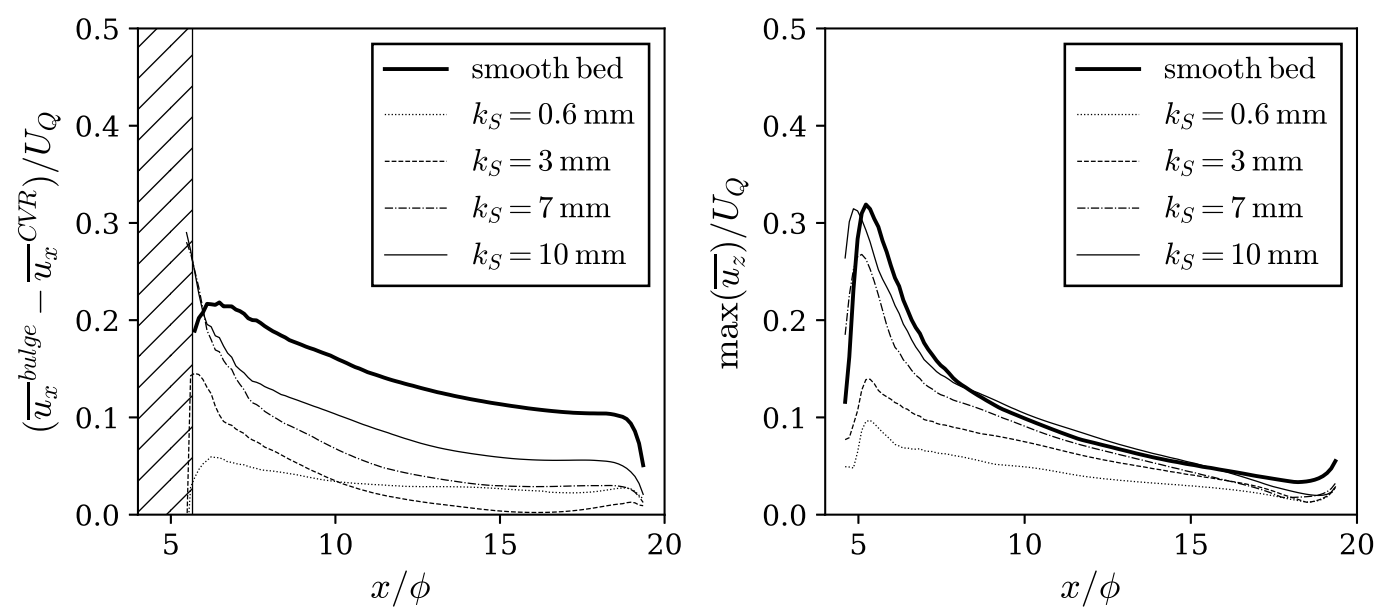

(b) $Q=131 \mathrm{~s}^{-1}$

FIG. 19. Longitudinal evolution of mean streamwise velocity-bulge magnitude (left) and upwash flow magnitude (right), observed in numerical simulations in the longitudinal plane, for various bed roughness conditions and flow rates $(a) 71 \mathrm{~s}^{-1}$ and $(b) 131 \mathrm{~s}^{-1}$. The velocity bulge is only defined outside of the reverse flow region (i.e $x / \phi>\max \left[l_{r}(z) / \phi\right]+4$ ) and the hatched rectangles represent the extent of the maximum reverse flow region observed for the different $k_{s}$.

Dupuis et al. ${ }^{5}$ observed a larger velocity-bulge for a smooth bed than for a rough bed. 


\section{Reverse flow region}

In the cylinder wake, the reverse flow region is defined as the area where mean streamwise velocity $\overline{u_{x}}$ is negative. The measured and simulated reversed flow regions are shown in Fig. 14 (bounded by black solid lines) for the four flow cases. The effect of the $k_{s}$-value on the vertical distribution of the length of the reversed flow region $l_{r}$ was observed in the two sets of numerical simulations presented in section VI B, and is displayed in Fig. 20.

Based on the measurements in Fig. 14, the depth-averaged length of the reverse flow region is larger for the rough-bed cases $(15 \mathrm{~mm}$ and $11 \mathrm{~mm}$ for Q13r and Q7r, respectively) than for the smooth-bed cases (6 mm), as observed by Sadeque, Rajaratnam, and Loewen ${ }^{25}$ for a single emergent circular cylinder. However, this result is not retrieved in the simulations (see also Fig. 14). As a result, it is not easy to draw a definite conclusion on the effect of the bed-surface state on the extent of the reverse flow region.

For a rough-bed flow case, measurements and numerical results (Figs. 14 and 20) indicate that, below $z / \phi \approx 2, l_{r}$ could decrease when approaching the channel bottom. This decrease is faster with a rougher bed (Fig. 20). This result could be compared to the results obtained for the transverse velocity fluctuations in Figs. 10 and 11. An increase in bed roughness leads to a higher bed-induced turbulence that strongly disorganizes the transverse fluctuating motion and the associated vortex shedding in the region $z / \phi \leq 2$. This bed stabilization effect on the coherent structures could have also an impact on the extent of the reverse flow region.

The previous results are in agreement with the observations of Heidari et al. ${ }^{43}$, who experimentally investigated the wake of a slender emergent cylinder $(A R=11)$ in a shallow open channel. They observed that the length of the reversed flow region behind the cylinder was smaller near the bed. In addition, an unsteady bubble regime was observed for the wake close to the bed and a vortex shedding regime was observed otherwise. The authors concluded that for high aspect ratios the bed stabilization effect should be limited to a near bed region.

Last, both experimental and numerical results (Figs. 14 and 20) show that the vertical $l_{r^{-}}$ distribution is independent of the shallowness level. 


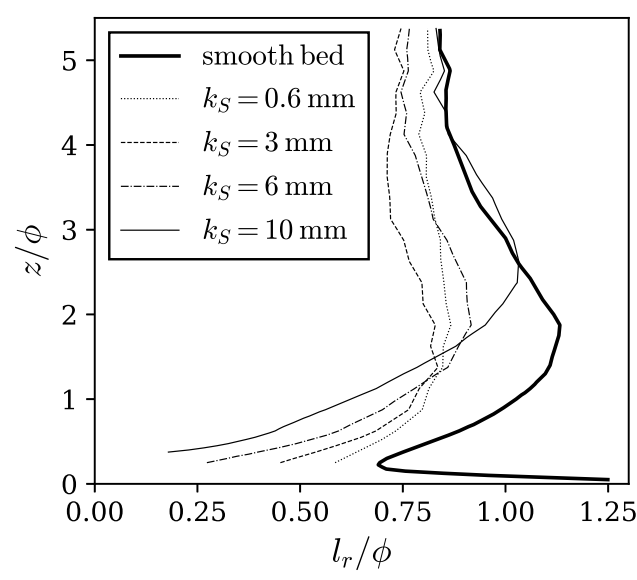

(a) $Q=71 \mathrm{~s}^{-1}$

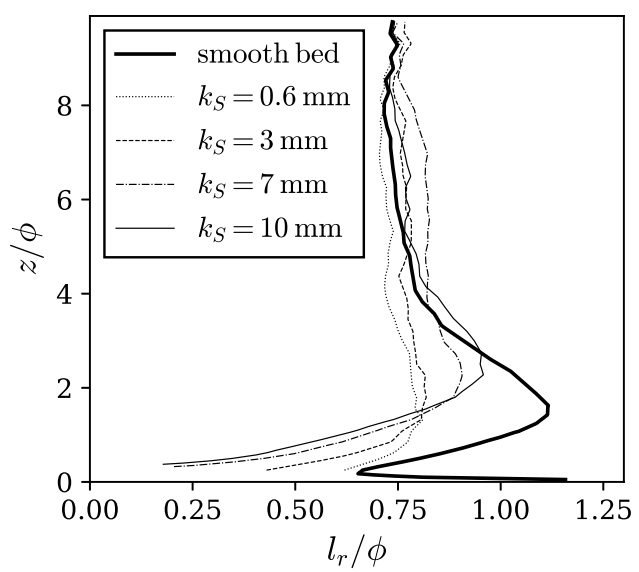

(b) $Q=131 \mathrm{~s}^{-1}$

FIG. 20. Extent of the reverse flow region measured from the downstream cylinder edge $l_{r}$ : simulations for a variable bed roughness heigth $k_{s}$ and flow rates $(a) 71 \mathrm{~s}^{-1}$ and $(b) 131 \mathrm{~s}^{-1}$.

\section{CONCLUSION}

Free-surface flows through a staggered cylinder array were investigated in an $18 \mathrm{~m}$ long and $1 \mathrm{~m}$ wide open-channel flume. The wooden circular cylinders simulated rigid emergent vegetation. Four flow cases were studied with a two-factor design comprising flow rate $\left(7\right.$ and $\left.131 \mathrm{~s}^{-1}\right)$ and bed-surface state (hydraulically-rough and -smooth). Transient flow simulations using the code OpenFOAM and a hybrid RANS-LES turbulence model were also carried out, to gain further insight into the effect of a variable bed roughness height on the flow structure.

The study was first and foremost aimed at assessing the relative and combined effects of the bed roughness and shallowness on the mean flow patterns and velocity fluctuations within the 
cylinder array. For all cases, both measurements and simulations highlighted an upward flow in the near-cylinder wake, with development of two counter-rotating secondary currents cells further downstream. The latter brought high-momentum fluid from the high-speed region into the cylinder wake, resulting in a near-bed streamwise velocity-bulge. Between two longitudinal cylinder rows, the secondary currents are also responsible for a flow deceleration, as low-momentum fluid from the cylinder wake is brought into the high-speed region. The measurements showed that upward flow and velocity-bulge were greatest for the rough-bed cases compared to the smooth-bed cases. The simulations also indicated that the upward flow and velocity-bulge increased with an increasing roughness height. The bed roughness effect could be similar to the effect of the approaching boundary layer thickness observed for a single cylinder ${ }^{15-18}$. An increase of the bed roughness would result in a stronger near-bed velocity gradient and in more bent von Karman vortices ${ }^{15}$, which in turn would give rise to stronger base vortices. Last, with an increase in the roughness height, the secondary currents decayed faster in the longitudinal direction. This could be ascribed to an increase of the bed-induced turbulence and of its inhibiting effect on the transverse fluctuating flow motion.

The results of the bed roughness and shallowness effects on the flow structure could be of interest in the context of bed morphodynamics. As both the near-bed flow acceleration and the magnitude of secondary currents increase with the height of the roughness elements, we might expect that scour depth behind rigid vegetation increases with the bed roughness, as well as the two erosion pits generated by the helical secondary currents. New experiments with a moveable bed should be required to confirm this assumption, as erosion processes would also have a retroactive effect on the vertical distribution of streamwise velocity, thus on the inclination of the von Karman vortices near the ground, and eventually on the secondary currents.

The secondary purpose of the study was to quantify the free-surface waves that are induced by the vortex shedding behind the cylinders. Three flow cases involve noticeable free-surface oscillations, termed 'seiche waves'. The latter are composed of a transverse standing wave and a longitudinal progressive wave moving upstream, both waves having the same frequency but different wave-lengths. The amplitude of the standing wave increases when moving downstream, reaching 5 to $8 \%$ of the flow depth. Based on the spectra of flow depth and transverse velocity fluctuations, two oscillation modes can be simultaneously excited (modes 6 and 7). A predominant mode, whose frequency can be measured at anti-nodes, controls the transverse distributions of flow depth fluctuations. Owing to the lock-in process between surface waves and vortex shedding, 
this predominant mode is observed in the spectral signature of the velocity fluctuations behind the cylinders. As observed by Viero, Pradella, and Defina ${ }^{42}$ for a staggered array of cylinders, a secondary resonance occurs at $S_{t}=0.16$. Last, a secondary mode, whose frequency can be measured at nodes, is responsible for the non-zero oscillations at these positions.

Both shallowness and bed roughness were found to have an impact on the seiche waves. For a given bed-surface state, the flow depth fluctuations associated to both modes decrease with an increasing shallowness. The decrease in the predominant mode fluctuations could be explained by a decreasing strength of the oscillating lift-force associated with vortex shedding, as this force is applied to a decreasing length of cylinder. For a given shallowness level, rough-bed-induced turbulence attenuated the secondary mode fluctuations by disorganizing the transverse fluctuating flow motion. For the smaller discharge over rough bed, even the predominant mode was attenuated, under the combined effects of a high rough-bed-induced turbulence and a small distance of application of the lift-force, resulting in the absence of seiche waves. The damping effect of the rough-bed-induced turbulence on the transverse fluctuating motion is visible in the near-bed region, but also higher in the water column where e.g. the region of high Reynolds stress is less wide for the rough-bed cases (compared to the smooth-bed cases), irrespective of the shallowness level.

This study also shows that free-surface oscillations have little effect on the mean flow patterns in the near-bed region (velocity-bulge and helical secondary currents). However, these oscillations could somehow impact the mean velocity magnitude and the turbulence intensities, as reflected by some discrepancies between experiments and simulations (based on a rigid lid assumption). A part of these discrepancies could also stem from the use of wall functions, which could lead to an underestimate of the bed-induced streamwise fluctuating motion.

\section{ACKNOWLEDGMENTS}

The authors wish to thank A. Buffet and F. Thollet for their technical support, and Céline Berni for our fruitful discussions. This research work was funded by the French National Research Agency under grant no. ANR-14-CE03-0010 (Flowres ANR project, https : //flowres.inrae . $\mathrm{fr} / \mathrm{en} /)$. 


\section{AUTHOR DECLARATIONS}

\section{A. Conflict of interest}

The authors have no conflicts to disclose.

\section{B. Data Availability}

The data that support the findings of this study are available from the corresponding author upon reasonable request.

\section{REFERENCES}

${ }^{1}$ H. M. Nepf and E. W. Koch, "Vertical secondary flows in submersed plant-like arrays," Limnology and Oceanography 44, 1072-1080 (1999), http://dx.doi.org/10.4319/10.1999.44. 4.1072.

${ }^{2}$ H. S. Kim, M. Nabi, I. Kimura, and Y. Shimizu, "Computational modeling of flow and morphodynamics through rigid-emergent vegetation," Advances in Water Resources 84, 64-86 (2015), http://dx.doi.org/10.1016/j.advwatres.2015.07.020.

${ }^{3}$ D. Liu, P. Diplas, J. D. Fairbanks, and C. C. Hodges, “An experimental study of flow through rigid vegetation," Journal of Geophysical Research 113 (2008), http://dx.doi.org/10. 1029/2008 JF001042.

${ }^{4}$ R. Martino, A. Paterson, and M. Piva, "Double-average mean flow and local turbulence intensity profiles from PIV measurements for an open channel flow with rigid vegetation,' Environmental Fluid Mechanics 12, 45-62 (2012), http: / /dx.doi .org/10 .1007/s10652-011-9221-4.

${ }^{5}$ V. Dupuis, S. Proust, C. Berni, and A. Paquier, "Combined effects of bed friction and emergent cylinder drag in open channel flow," Environmental Fluid Mechanics 16, 1173-1193 (2016), http://dx.doi.org/10.1007/s10652-016-9471-2.

${ }^{6}$ H. M. Nepf, “Drag, turbulence, and diffusion in flow through emergent vegetation,” Water Resources Research 35, 479-489 (1999), http: //dx. doi .org/10. 1029/1998WR900069.

${ }^{7}$ Y. Tanino and H. M. Nepf, "Laboratory Investigation of Mean Drag in a Random Array of Rigid, Emergent Cylinders," Journal of Hydraulic Engineering 134, 34-41 (2008), http://dx.doi . org/10.1061/ (ASCE) 0733-9429(2008) 134:1(34). 
${ }^{8}$ A. M. Ricardo, K. Koll, M. J. Franca, A. J. Schleiss, and R. M. L. Ferreira, "The terms of turbulent kinetic energy budget within random arrays of emergent cylinders," Water Resources Research 50, 4131-4148 (2014), http: //dx.doi .org/10.1002/2013WR014596.

${ }^{9}$ B. L. White and H. M. Nepf, "Shear instability and coherent structures in shallow flow adjacent to a porous layer," Journal of Fluid Mechanics 593 (2007), http://dx.doi .org/10.1017/ S0022112007008415.

${ }^{10}$ L. Zong and H. Nepf, "Vortex development behind a finite porous obstruction in a channel," Journal of Fluid Mechanics 691, 368-391 (2012), http: //dx.doi .org/10.1017/jfm. 2011. 479.

${ }^{11}$ D. Liu, P. Diplas, C. Hodges, and J. Fairbanks, "Hydrodynamics of flow through double layer rigid vegetation," Geomorphology 116, 286-296 (2010), http://dx.doi.org/10.1016/j . geomorph.2009.11.024.

${ }^{12}$ T. Stoesser, G. P. Salvador, W. Rodi, and P. Diplas, "Large Eddy Simulation of Turbulent Flow Through Submerged Vegetation," Transport in Porous Media 78, 347-365 (2009), http: //dx . doi.org/10.1007/s11242-009-9371-8.

${ }^{13}$ T. Stoesser, S. J. Kim, and P. Diplas, "Turbulent Flow through Idealized Emergent Vegetation," Journal of Hydraulic Engineering 136, 1003-1017 (2010), http: //dx.doi .org/10. 1061/ (ASCE) HY.1943-7900.0000153.

${ }^{14}$ D. Sumner, J. L. Heseltine, and O. J. P. Dansereau, "Wake structure of a finite circular cylinder of small aspect ratio," Experiments in Fluids 37, 720-730 (2004), http://dx.doi .org/10. $1007 / \mathrm{s} 00348-004-0862-7$.

${ }^{15}$ H. F. Wang and Y. Zhou, "The finite-length square cylinder near wake," Journal of Fluid Mechanics 638, 453 (2009), http: //dx.doi.org/10.1017/S0022112009990693.

${ }^{16}$ H. F. Wang, Y. Zhou, C. K. Chan, and K. S. Lam, "Effect of initial conditions on interaction between a boundary layer and a wall-mounted finite-length-cylinder wake," Physics of Fluids 18, 065106 (2006), http: //dx.doi.org/10.1063/1.2212329.

${ }^{17}$ Z. Hosseini, J. Bourgeois, and R. J. Martinuzzi, "Large-scale structures in dipole and quadrupole wakes of a wall-mounted finite rectangular cylinder," Experiments in Fluids 54 (2013), http: //dx.doi.org/10.1007/s00348-013-1595-2.

${ }^{18}$ M. El Hassan, J. Bourgeois, and R. Martinuzzi, "Boundary layer effect on the vortex shedding of wall-mounted rectangular cylinder,' Experiments in Fluids 56 (2015), http: / /dx . doi .org/ $10.1007 / \mathrm{s} 00348-014-1882-6$. 
${ }^{19}$ L. Chagot, F. Moulin, and O. Eiff, “Towards converged statistics in three-dimensional canopydominated flows," Experiments in Fluids 61 (2020), 10.1007/s00348-019-2857-4.

${ }^{20}$ A. Monti, M. Omidyeganeh, and A. Pinelli, "Large-eddy simulation of an open-channel flow bounded by a semi-dense rigid filamentous canopy: Scaling and flow structure," Physics of Fluids 31, 065108 (2019), https://doi.org/10.1063/1.5095770.

${ }^{21}$ M. Adaramola, O. Akinlade, D. Sumner, D. Bergstrom, and A. Schenstead, "Turbulent wake of a finite circular cylinder of small aspect ratio," Journal of Fluids and Structures 22, 919-928 (2006), http://dx.doi.org/10.1016/j.jfluidstructs.2006.04.007.

${ }^{22}$ S. Tanaka and S. Murata, "An Investigation of the Wake Structure and Aerodynamic Characteristics of a Finite Circular Cylinder. Time-Averaged Wake Structures behind Circular Cylinders with Various Aspect Ratios.” JSME International Journal Series B 42, 178-187 (1999), http://dx.doi.org/10.1299/jsmeb.42.178.

${ }^{23}$ O. Frederich, E. Wassen, F. Thiele, M. Jensch, M. Brede, F. Hüttmann, and A. Leder, "Numerical simulation of the flow around a finite cylinder with ground plate in comparison to experimental measurements," in New Results in Numerical and Experimental Fluid Mechanics VI (Springer, 2007) pp. 348-355, http: //dx.doi .org/10.1007/978-3-540-74460-3_43.

${ }^{24}$ S. Krajnović, "Flow around a tall finite cylinder explored by large eddy simulation," Journal of Fluid Mechanics 676, 294-317 (2011), http: //dx.doi .org/10.1017/S0022112011000450.

${ }^{25}$ M. F. Sadeque, N. Rajaratnam, and M. R. Loewen, "Shallow turbulent wakes behind bedmounted cylinders in open channels," Journal of Hydraulic Research 47, 727-743 (2009), http: //dx.doi.org/10.3826/jhr.2009.3464.

${ }^{26}$ D. Chen and G. H. Jirka, "Experimental study of plane turbulent wakes in a shallow water layer," Fluid Dynamics Research 16, 11-41 (1995), http://dx.doi.org/10.1016/0169-5983(95) 00053-G.

${ }^{27}$ R. G. Ingram and V. H. Chu, "Flow around islands in Rupert Bay: An investigation of the bottom friction effect," Journal of Geophysical Research 92, 14521 (1987), http: //dx.doi .org/10. 1029/JC092iC13p14521.

${ }^{28}$ L. Zima and N. Ackermann, "Wave Generation in Open Channels by Vortex Shedding from Channel Obstructions," Journal of Hydraulic Engineering 128, 596-603 (2002), http: //dx . doi .org/10.1061/(ASCE)0733-9429(2002)128:6(596).

${ }^{29}$ A. Sarkar, "Vortex-Excited Transverse Surface Waves in an Array of Randomly Placed Circular Cylinders," Journal of Hydraulic Engineering 138, 610-618 (2012), http: //dx . doi .org/10 . 
1061/ (ASCE) HY.1943-7900.0000557.

${ }^{30}$ A. Defina and I. Pradella, "Vortex-induced cross-flow seiching in cylinder arrays," Advances in Water Resources 71, 140-148 (2014), http://dx.doi.org/10.1016/j.advwatres. 2014. 06.002.

${ }^{31}$ K. Zhao, N. Cheng, and Z. Huang, "Experimental study of free-surface fluctuations in openchannel flow in the presence of periodic cylinder arrays," Journal of Hydraulic Research 52, 465-475 (2014), http://dx.doi.org/10.1080/00221686.2014.880858.

${ }^{32}$ S. J. McLelland and A. P. Nicholas, "A new method for evaluating errors in high-frequency ADV measurements," Hydrological Processes 14, 351-366 (2000), http://dx.doi .org/10.1002/ (SICI) 1099-1085(20000215) $14: 2<351:$ : AID-HYP963>3 . 0 . CO ; 2-K.

${ }^{33}$ D. G. Goring and V. I. Nikora, "Despiking Acoustic Doppler Velocimeter Data," Journal of Hydraulic Engineering 128, 117-126 (2002), http://dx.doi.org/10.1061/(ASCE) 0733-9429(2002) 128:1(117).

${ }^{34}$ S. Proust and V. I. Nikora, "Compound open-channel flows: effects of transverse currents on the flow structure," Journal of Fluid Mechanics 885 (2020), https : //doi . org/10 .1017/jfm. 2019.973.

${ }^{35}$ Y. Peltier, N. Rivière, S. Proust, E. Mignot, A. Paquier, and K. Shiono, "Estimation of the error on the mean velocity and on the Reynolds stress due to a misoriented ADV probe in the horizontal plane: Case of experiments in a compound open-channel," Flow Measurement and Instrumentation 34, 34-41 (2013), http://dx.doi.org/10.1016/j.flowmeasinst.2013. 08.002.

${ }^{36}$ Y. Egorov and F. Menter, "Development and Application of SST-SAS Turbulence Model in the DESIDER Project," in Advances in Hybrid RANS-LES Modelling, Vol. 97 (Springer Berlin Heidelberg, 2008) pp. 261-270, https : //doi .org/10 .1007/978-3-540-77815-8_27.

${ }^{37}$ L. H. Yu, J. Zhan, and Y. Li, "Numerical investigation of drag force on flow through circular array of cylinders," Journal of Hydrodynamics, Ser. B 25, 330-338 (2013), http: //dx . doi . org/10.1016/S1001-6058(11)60371-6.

${ }^{38}$ F. R. Menter, “Two-equation eddy-viscosity turbulence models for engineering applications," AIAA Journal 32, 1598-1605 (1994), https : //doi .org/10. 2514/3.12149.

${ }^{39} \mathrm{~V}$. Dupuis, Experimental investigation of flows subjected to a longitudinal transition in hydraulic roughness in single and compound channels, Ph.D. thesis, Université de Lyon (2016), https: //tel.archives-ouvertes.fr/tel-01409745. 
${ }^{40}$ M. Chetibi, S. Proust, and S. Benmamar, "Transverse surface waves in steady uniform and non-uniform flows through an array of emergent and slightly submerged square cylinders," Journal of Hydraulic Research 58, 605-617 (2020), https ://doi .org/10 .1080/00221686. 2019.1647885 .

${ }^{41}$ R. G. Dean and R. A. Dalrymple, Water Wave Mechanics for Engineers and Scientists, Advanced Series on Ocean Engineering, Vol. 2 (World Scientific, 1991) http://dx.doi .org/10.1142/ 1232.

${ }^{42}$ D. P. Viero, I. Pradella, and A. Defina, "Free surface waves induced by vortex shedding in cylinder arrays," Journal of Hydraulic Research 55, 16-26 (2017), https://doi.org/10.1080/00221686.2016.1217948.

${ }^{43}$ M. Heidari, R. Balachandar, V. Roussinova, and R. M. Barron, "Characteristics of flow past a slender, emergent cylinder in shallow open channels," Physics of Fluids 29, 065111 (2017), http://dx.doi.org/10.1063/1.4986563.

${ }^{44}$ M. Chatelain and S. Proust, "Nonuniform flows in a compound open-channel: assessment of a hybrid rans-les approach,” Water Resources Research 56 (2020), https : / doi .org/10 . 1029/ 2020 WR02705.

${ }^{45}$ A. Roshko, "Experiments on the flow past a circular cylinder at very high Reynolds number," Journal of Fluid Mechanics 10, 345 (1961), http://dx.doi.org/10.1017/ S0022112061000950.

${ }^{46}$ W. Huai, Z. Wu, Z. Qian, and C. Geng, "Large Eddy Simulation of open channel flows with non-submerged vegetation,” Journal of Hydrodynamics, Ser. B 23, 258-264 (2011), http: // dx.doi .org/10.1016/S1001-6058(10)60111-4. 